This report was prepared as an account of work sponsored by an agency of the United States Government. Neither the United States Government nor any agency thereof, nor any of their employees, makes any warranty, express or implied, or assumes any legal liability or responsibility for the accuracy, completeness, or usefulness of any information, apparatus, product, or process disclosed, or represents that its use would not infringe privately owned rights. Reference herein to any specific commercial product, process, or service by trade name, trademark, manufacturer, or otherwise does not necessarily constitute or imply its endorsement, recommendation, or favoring by the United States Government or any agency thereof. The views and opinions of authors expressed herein do not necessarily state or reflect those of the United States Government or any agency thereof.

\title{
SURVEY OF THERMAL-HYDRAULIC MODELS OF COMMERCIAL NUCLEAR POWER PLANTS
}

\author{
J. C. Determan \\ C. E. Hendrix
}

Published December 1992

\section{Idaho National Engineering Laboratory \\ EG\&G Idaho, Inc. Idaho Falls, Idaho 83415}

\author{
Prepared for the \\ Division of Reactor Systems Safety \\ Office of Nuclear Regulatory Research \\ U.S. Nuclear Regulatory Commission \\ Washington, D.C. 20555 \\ Under DOE Idaho Fleld Otfice \\ Contract DE-AC07-761D01570
}

FIN A6328 


\section{ABSTRACT}

A survey of existing nuclear power plant decks has been performed; data collecteo for decks at the Idaho National Engineering Laboratory, Los Alamos National Laboratory, and Code Applications and Maintenance Program members are presented. A PC based database used to hold the data is described. Recommendations for improvement of the NRC's analysis capability are presented.

FIN No. A6328-Thermal-Hydraulic Technical Support Center 


\section{CONTENTS}

ABSTRACT $\ldots \ldots \ldots \ldots \ldots \ldots \ldots \ldots \ldots \ldots \ldots \ldots \ldots \ldots \ldots \ldots \ldots \ldots \ldots \ldots \ldots \ldots$

SUMMARY $\ldots \ldots \ldots \ldots \ldots \ldots \ldots \ldots \ldots \ldots \ldots \ldots \ldots \ldots \ldots \ldots \ldots \ldots$

ACKNOWLEDGEMENTS $\ldots \ldots \ldots \ldots \ldots \ldots \ldots \ldots \ldots \ldots \ldots \ldots \ldots \ldots \ldots$ ix

1. INTRODUCTION $\ldots \ldots \ldots \ldots \ldots \ldots \ldots \ldots \ldots \ldots \ldots \ldots \ldots \ldots \ldots \ldots \ldots \ldots$

2. GROUP CLASSIFICATION OF DOMESTIC LWRS $\ldots \ldots \ldots \ldots \ldots \ldots \ldots \ldots$

3. LIST OF THERMAL-HYDRAULIC MODELS $\ldots \ldots \ldots \ldots \ldots \ldots \ldots \ldots \ldots$

4. PC BASED DATABASE $\ldots \ldots \ldots \ldots \ldots \ldots \ldots \ldots \ldots \ldots \ldots \ldots \ldots \ldots \ldots$

5. REFERENCES $\ldots \ldots \ldots \ldots \ldots \ldots \ldots \ldots \ldots \ldots \ldots \ldots \ldots \ldots \ldots \ldots \ldots \ldots$

Appendix A-Data Gathered in the Survey

Appendix B-Using the Database Programs

\section{TABLES}

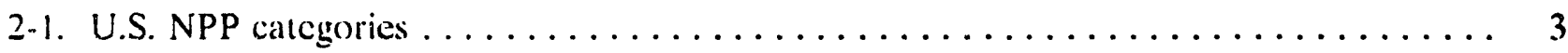

2-2. CAMP member NPPs in survey $\ldots \ldots \ldots \ldots \ldots \ldots \ldots \ldots \ldots \ldots \ldots \ldots \ldots \ldots \ldots \ldots \ldots$

3-1. List of thermal-hydraulic input decks $\ldots \ldots \ldots \ldots \ldots \ldots \ldots \ldots \ldots \ldots$

3-2. Input deck applications matrix $\ldots \ldots \ldots \ldots \ldots \ldots \ldots \ldots \ldots \ldots \ldots \ldots \ldots$ 


\section{SUMMARY}

A survey of the thermal-hydraulic models of nuclear power plants has been performed to identify the NRC's current analytical capabilities for critical event response. The survey also supports ongoing research for accident management.

The results of the survey are presented here. The PC database which records detailed data on each model is described. 


\section{ACKNOWLEDGEMENTS}

The authors of this report would like to thank Don Fletcher, Dick Schultz, Ron Beelman, Phil Wheatley, John Burtt, Bob Hanson, Len Ward, Chuck

Dobbe, and Paul Bayless for their input to this document. 


\section{Survey of Thermal-Hydraulic Models of Commercial Nuclear Power Plants}

\section{INTRODUCTION}

The NRC's response to critical events and its support of ongoing research depend on their current thermal-hydraulic modeling capability. Knowledge of the existence and capabilities of input decks of domestic light water reactors (LWRs) could greatly accelerate the NRC's analysis of critical events. This knowledge also helps identify the strengths and weaknesses of the NRC's present modeling capabilities.

The purpose of this report is to document the existence of nuclear power plant (NPP) input decks and to identify possible enhancements to the NRC's NPP modeling capability. This report is intended as a living document. Decks from the Idaho National Engineering Laboratory (INEL), Los Alamos National Laboratory (LANL), and the code applications and maintenance program (CAMP) members are currently incorporated. Methods for enhancing the NRC's modeling capability are presented. These suggested improvements are focused on the NRC's capability for modeling LWR systems with the RELAP5, TRAC-PF1, and TRAC-BD1 codes. A system for classifying the existing domestic LWRs into distinct groups of similar type, based on the August 28, 1980 letter by J. A. Dearien, ${ }^{1}$ is presented as an aid to identifying the enhancements noted above.

Section 2 presents the LWR classification system. The input decks currently identified are listed in Section 3. Section 4 describes the PC based database used to store the data.

Appendix A presents the data for each plant currently included in the survey. Appendix B describes the use of the database and its associated programs. 


\section{GROUP CLASSIFICATION OF DOMESTIC LWRS}

Domestic commercial LWRs fall into two broad categories: pressurized water reactors (PWR) or boiling water reactors (BWR). Domestic LWRs may be further classified by vendor. The BW/Rs are produced by General Electric (GE), and the PWRs are produced by Westinghouse (W), Combustion Engineering $(C E)$, and Babcock and Wilcox $(B \& W)$. Each vendor has produced different reactor designs over the years, varying such things as the core size and coolant loop arrangement. LWRs with a common vendor and reactor design may be considered similar from a thermal-hydraulic modeling standpoint. A thermal-hydraulic model of one LWR may be applied to any similar LWR with only minor modifications to the model. Therefore, classifying domestic LWRs indicates the minimum set of thermal-hydraulic models needed to allow analysis of incidents at any domestic LWR quickly.

Domestic LWRs have been classified by J. A. Dearien ${ }^{[1]}$; this system was modified to account for differences in fuel bundle design, and to correct errors in the original list ${ }^{[2]}$. The principal parameters considered in grouping BWRs include: 1) nuclear steam supply system (NSSS) design; 2) number of fuel bundles; 3) vessel ID; 4) fuel bundle design; and, 5) containment design. The principal parameters considered in grouping PWRs include: 1) reactor vendor; 2) coolant loop design; 3) number of fuel bundles; 4) fuel bundle design; and, 5) containment design (Westinghouse PWRs only). Thermal power was not considered in grouping the plants because it depends primarily upon the number and design of the fuel bundles, and would be redundant. Table 2-1 lists the U.S. NPPs by group, states the distinguishing characteristics of each group, and indicates individual differences possessed by plants within the group. Table $2-2$ provides the same information on foreign NPPs for which thermal-hydraulic input decks are available through CAMP. Comparing the entries in Table 2-1 will allow the analyst to estimate the level of effort needed to modify an input deck to represent a different plant in the same or a similar group. 


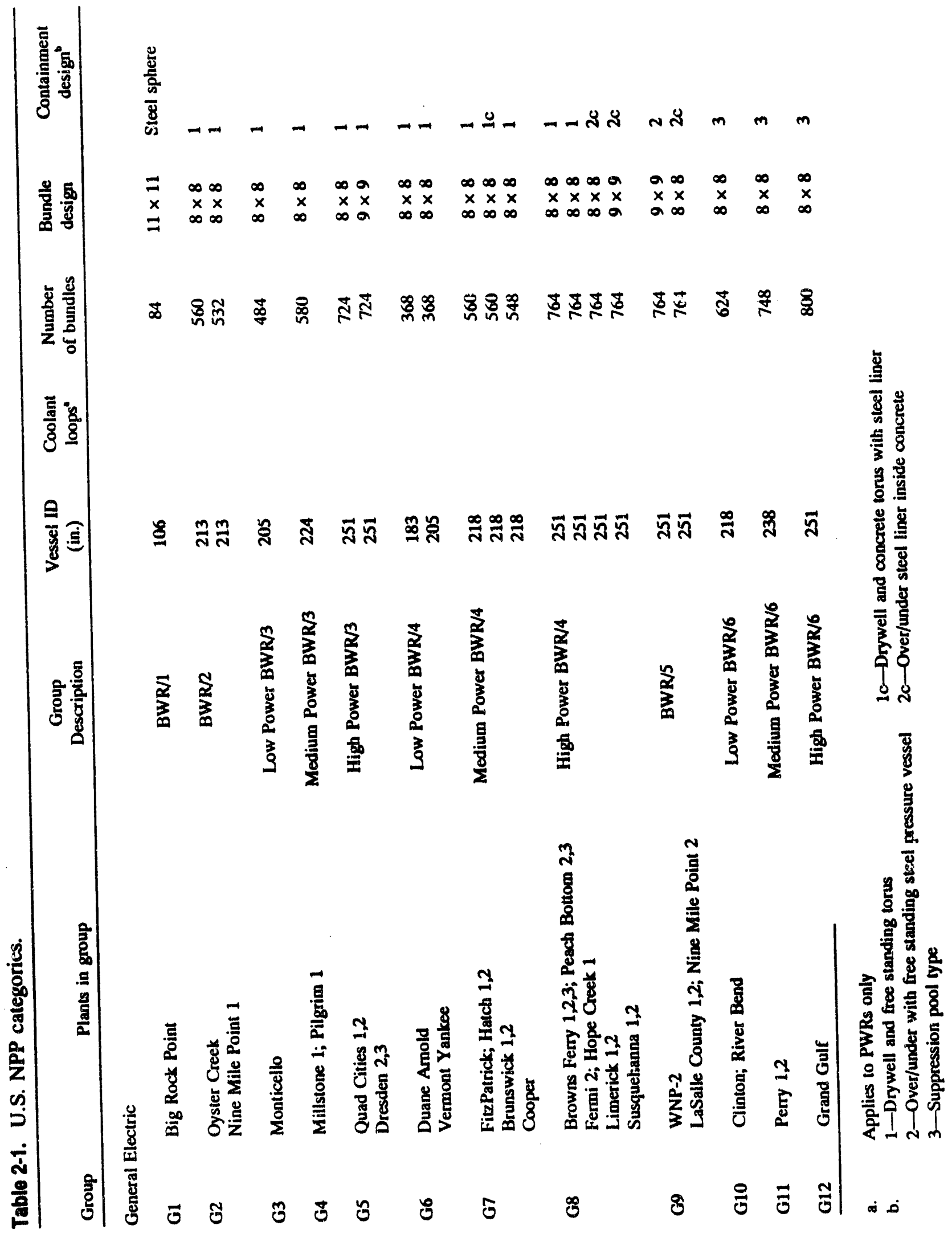




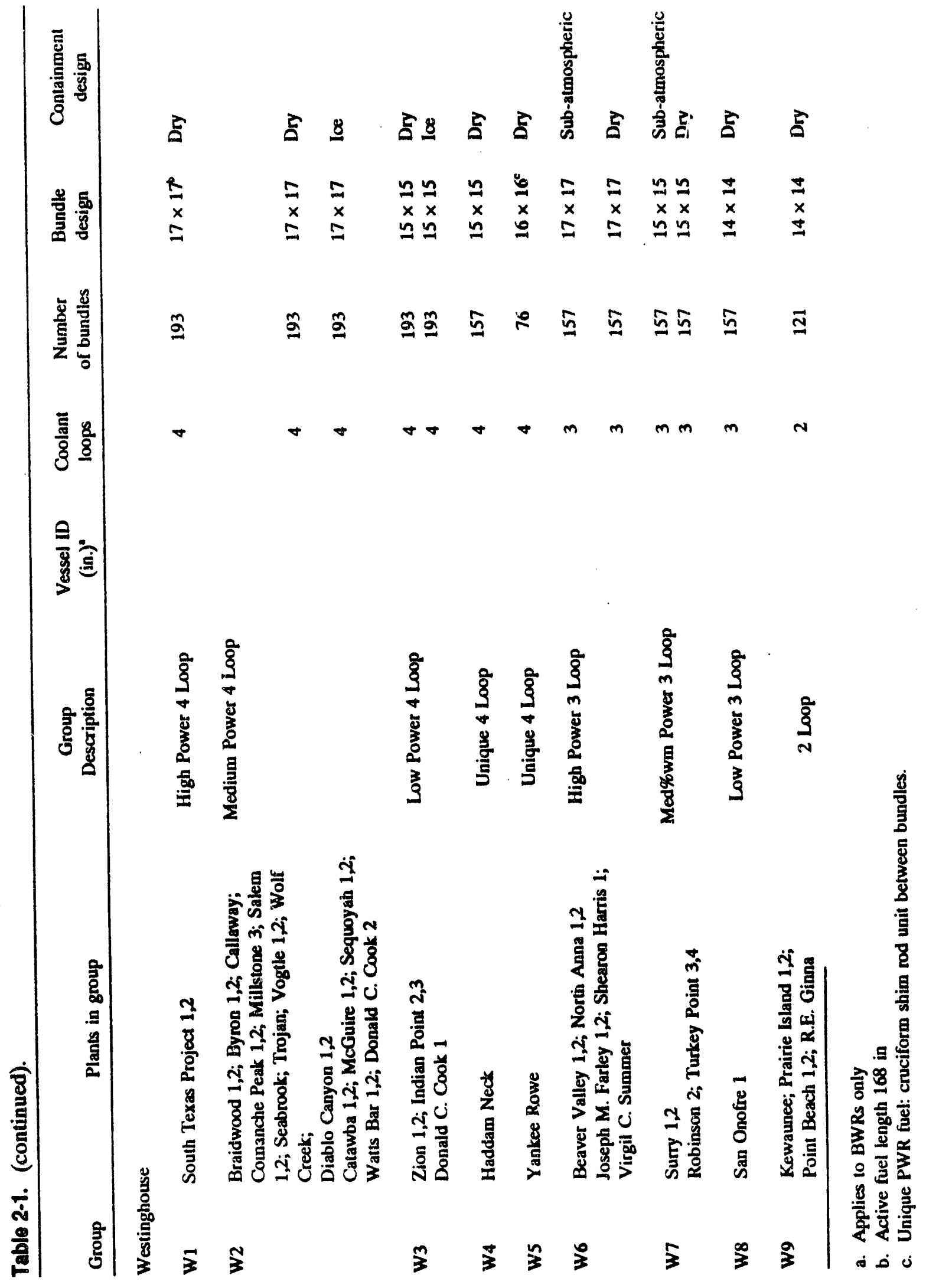




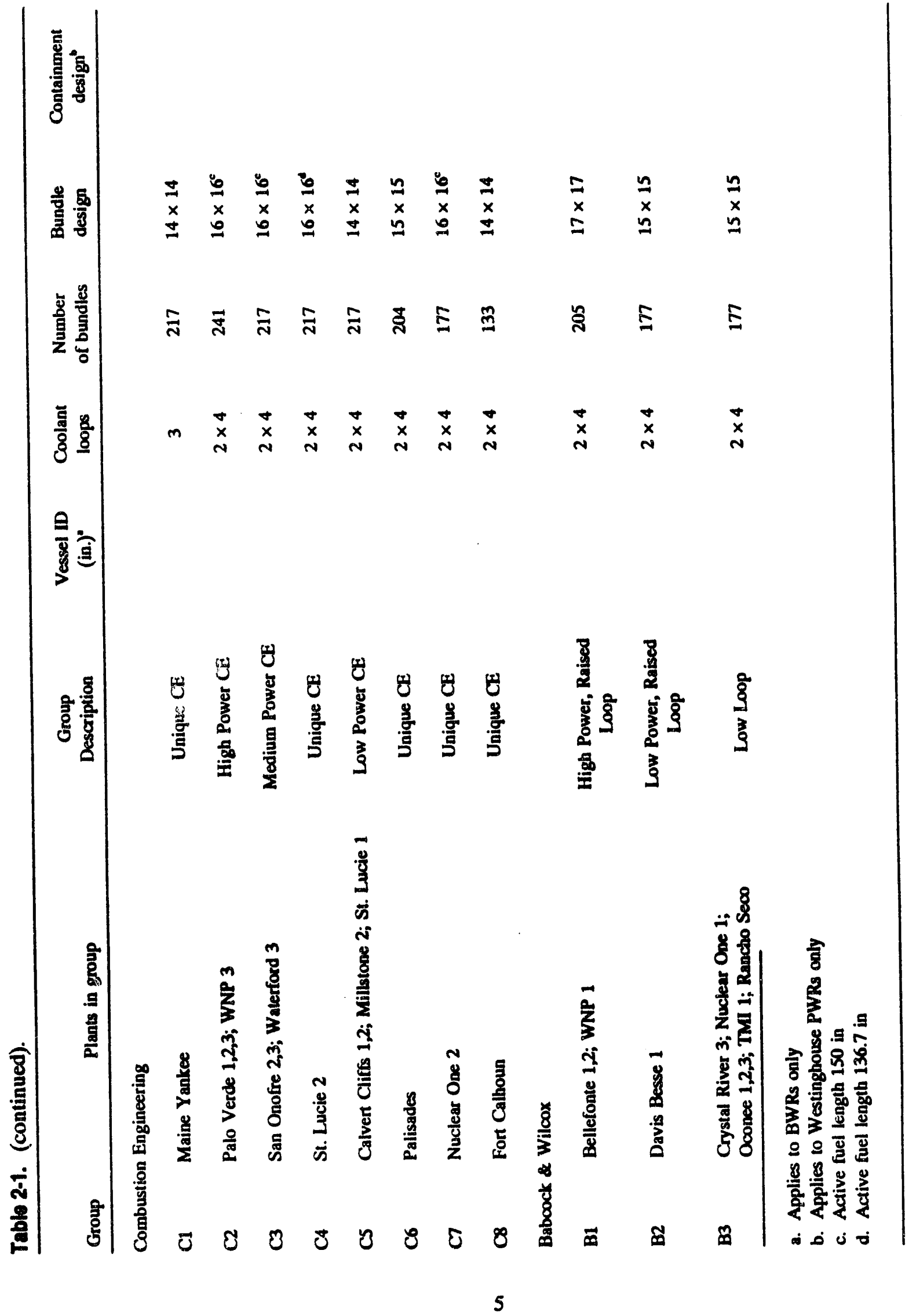




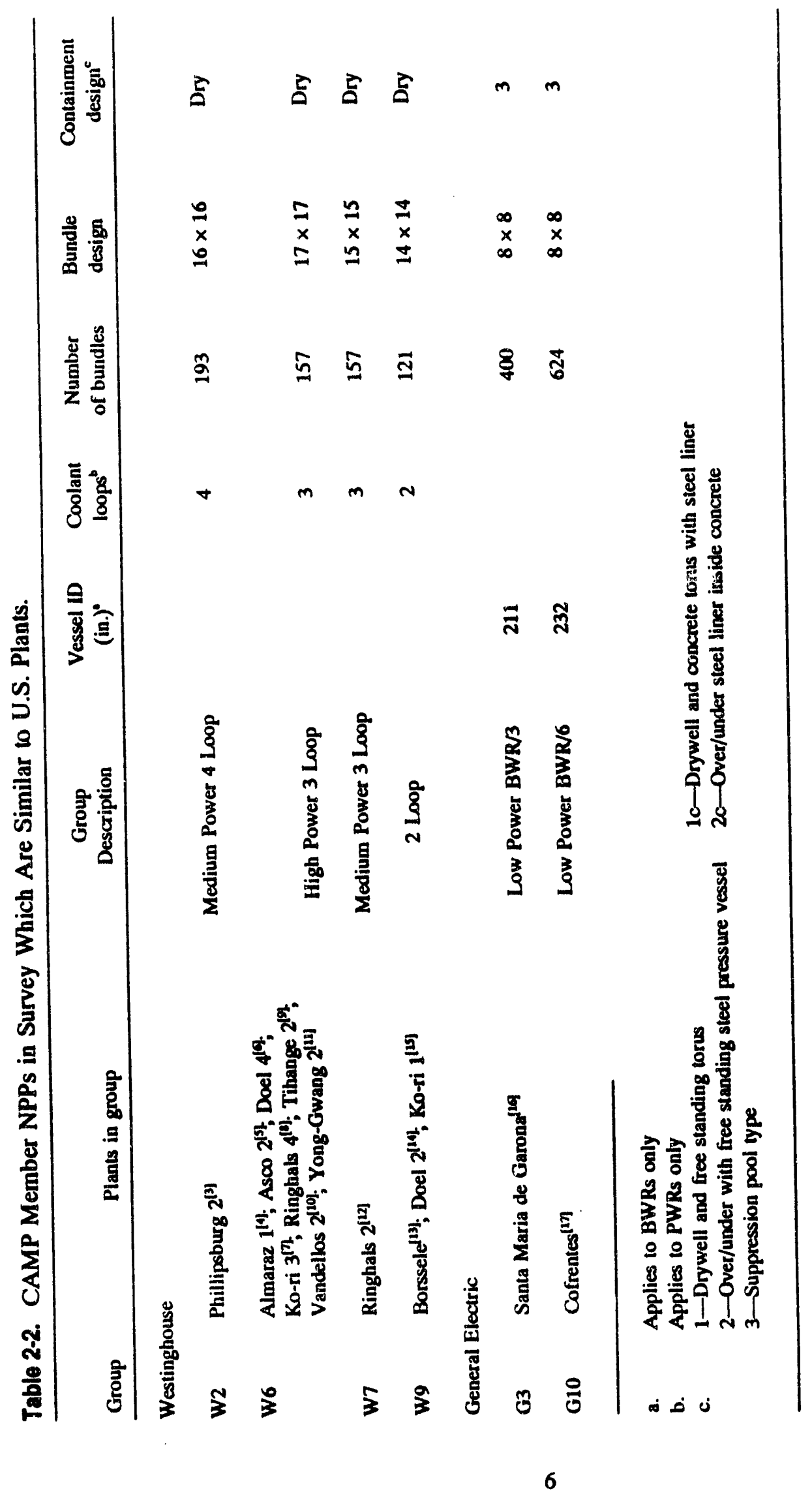




\section{LIST OF THERMAL-HYDRAULIC MODELS}

A search was made to identify the LWR systems that have been modeled with the commonly used thermal-hydraulic analysis codes. A summary giving the plant name, plant group, and model applications is presented here for each deck. Detailed information for each model, as recorded in the PC data base, is given in Appendix A.

Table 3-1 lists the thermal-hydraulic plant input decks identified in the survey. The input decks are grouped by analysis code and listed alphabetically within each group. The plant group from Table 2-1, the code version, and the custodian organization are listed for each input deck. Input decks frequently contain data considered proprietary to the vendor or utility of the plant being modeled, therefore the proprietary status of each deck is indicated in the table.

Table 3-2 is a matrix of input deck applications. Seventeen common reactor transients are listed along the top of the matrix and the input decks are listed alphabetically along the left edge of the matrix. An entry of " $A$ " in the matrix indicates an assessment or application of the input deck. There are no reported applications of the CESSAR RELAP5/MOD1, Generic B\&W TRAC-PF1/MOD1, Grand Gulf TRAC-BD1, or Robinson 2 RELAP5/MOD3 input decks. 
Table 3-1. List of thermal-hydraulic input decks.

\begin{tabular}{|c|c|c|c|c|}
\hline Plant & Group & Code version & Proprietary & Source \\
\hline \multicolumn{5}{|l|}{ RELAPS: } \\
\hline $\begin{array}{l}\text { Almaraz 1 } \\
\text { Borssele } \\
\text { Browns Ferry } 1 \\
\text { Calveri Cliffs } 1 \\
\text { CESSAR } \\
\text { Davis-Besse } \\
\text { Doel 2 } \\
\text { Doel } 4 \\
\text { Ko-ri } 1 \\
\text { Ko-ri } 3 \\
\text { North Anna } \\
\text { Nuclear Or e } 2 \\
\text { Ooonee 1 } \\
\text { Phillipsburg } 2 \\
\text { RESAR 3S } \\
\text { Robinson } 2 \\
\text { Robinson } 2 \\
\text { Tihange 2 } \\
\text { Vandellos } 2 \\
\text { Yankee Rowe } \\
\text { Yong-Gwang } 2 \\
\text { Zion 1 }\end{array}$ & $\begin{array}{l}\text { W6 } \\
\text { W9 } \\
\text { G8 } \\
\text { C5 } \\
\text { C2 } \\
\text { B2 } \\
\text { W9 } \\
\text { W6 } \\
\text { W9 } \\
\text { W6 } \\
\text { W6 } \\
\text { C7 } \\
\text { B3 } \\
\text { W2 } \\
\text { W2 } \\
\text { W7 } \\
\text { W7 } \\
\text { W6 } \\
\text { W6 } \\
\text { W5 } \\
\text { W6 } \\
\text { W3 }\end{array}$ & $\begin{array}{l}\text { RELAP5/MOD2 } \\
\text { RELAP5/MOD2 } \\
\text { RELAP5/MOD2 } \\
\text { RELAP5/MOD2 } \\
\text { RELAP5/MOD1 } \\
\text { RELAP5/MOD2 } \\
\text { RELAP5/MOD2 } \\
\text { RELAP5/MOD2 } \\
\text { RELAP5/MOD2 } \\
\text { RELAP5/MOD3 } \\
\text { RELAP5/MOD2 } \\
\text { RELAP5/MOD1.5 } \\
\text { RELAP5/MOD2 } \\
\text { RELAP5/MOD2 } \\
\text { RELAP5/MOD2 } \\
\text { RELAP5/MOD2 } \\
\text { RELAP5/MOD3 } \\
\text { RELAP5/MOD2 } \\
\text { RELAP5/MOD2 } \\
\text { RELAP5/MOD3 } \\
\text { RELAP5/MOD2 } \\
\text { RELAP5/MOD2 }\end{array}$ & $\begin{array}{l}\text { Yes } \\
\text { Yes } \\
\text { Yes } \\
\text { Yes } \\
\text { Yes } \\
\text { Yes } \\
\text { No } \\
\text { No } \\
\text { No } \\
\text { Yes } \\
\text { Yes } \\
\text { Yes } \\
\text { Yes } \\
\text { Yes } \\
\text { Yes } \\
\text { Yes } \\
\text { Yes } \\
\text { Yes } \\
\text { Yes } \\
\text { Yes } \\
\text { No } \\
\text { Yes }\end{array}$ & $\begin{array}{l}\text { CAMP } \\
\text { CAMP } \\
\text { INEL } \\
\text { INEL } \\
\text { INEL } \\
\text { INEL } \\
\text { CAMP } \\
\text { CAMP } \\
\text { CAMP } \\
\text { CAMP } \\
\text { INEL } \\
\text { INEL } \\
\text { INEL } \\
\text { CAMP } \\
\text { INEL } \\
\text { INEL } \\
\text { INEL } \\
\text { CAMP } \\
\text { CAMP } \\
\text { INEL } \\
\text { CAMP } \\
\text { INEL }\end{array}$ \\
\hline \multicolumn{5}{|l|}{ RELAPS/SCDAP: } \\
\hline $\begin{array}{l}\text { Bellefonte } 1,2 \\
\text { Calvert Cliffs } 1 \\
\text { Oconee } 1 \\
\text { Seabrook } \\
\text { Surry } 1\end{array}$ & $\begin{array}{l}\text { B1 } \\
\text { C5 } \\
\text { B3 } \\
\text { W7 }\end{array}$ & $\begin{array}{l}\text { RELAP5/MOD2/SCDAP } \\
\text { RELAP5/MOD3/SCDAP } \\
\text { RELAP5/MOD3/SCDAP } \\
\text { RELAP5/MOD3/SCDAP } \\
\text { RELAP5/MOD2/SCDAP }\end{array}$ & $\begin{array}{l}\text { No } \\
\text { Yes } \\
\text { Yes } \\
\text { Yes } \\
\text { Yes }\end{array}$ & $\begin{array}{l}\text { INEL } \\
\text { INEL } \\
\text { INEL } \\
\text { INEL } \\
\text { INEL }\end{array}$ \\
\hline \multicolumn{5}{|l|}{ TRAC-P: } \\
\hline $\begin{array}{l}\text { Asco } \\
\text { Calvert Cliffs 1 } \\
\text { Davis-Besse } \\
\text { Generic B\&W PWR } \\
\text { Oconee 1 } \\
\text { R.E. Ginna } \\
\text { RESAR 3S } \\
\text { Ringhals } 2 \\
\text { Ringhals } 4 \\
\text { Robirson } 2 \\
\text { TMI 1 } \\
\text { Vandellos } 2 \\
\text { Zion 1 }\end{array}$ & $\begin{array}{l}\text { W6 } \\
\text { C5 } \\
\text { B2 } \\
\text { B3 } \\
\text { W9 } \\
\text { W2 } \\
\text { W7 } \\
\text { W6 } \\
\text { W7 } \\
\text { B3 } \\
\text { W6 } \\
\text { W3 }\end{array}$ & $\begin{array}{l}\text { TRAC-PF1MOD1 } \\
\text { TRAC-PF1MOD1 } \\
\text { TRAC-PF1MOD1 } \\
\text { TRAC-PF1MOD1 } \\
\text { TRAC-PF1MOD1 } \\
\text { TRAC-PF1MOD1 } \\
\text { TRAC-PF1MOD1 } \\
\text { TRAC-PF1MOD1 } \\
\text { TRAC-PF1MOD1 } \\
\text { TRAC-PF1MOD1 } \\
\text { TRAC-PF1MOD1 } \\
\text { TRAC-PF1MOD1 } \\
\text { TRAC-PF1MOD1 }\end{array}$ & $\begin{array}{l}\text { Yes } \\
\text { Yes } \\
\text { Yes } \\
\text { Yes } \\
\text { Yes } \\
\text { Yes } \\
\text { Yes } \\
\text { Yes } \\
\text { Yes } \\
\text { Yes } \\
\text { Yes } \\
\text { Yes } \\
\text { Yes }\end{array}$ & $\begin{array}{l}\text { CAMP } \\
\text { LANL } \\
\text { LANL } \\
\text { LANL } \\
\text { LANL } \\
\text { LANL } \\
\text { LANL } \\
\text { CAMP } \\
\text { CAMP } \\
\text { LANL } \\
\text { LANL } \\
\text { CAMP } \\
\text { LANL }\end{array}$ \\
\hline \multicolumn{5}{|l|}{ TRAC.B: } \\
\hline $\begin{array}{l}\text { Black Fox } \\
\text { Browns Ferry } 1 \\
\text { BWR/6-218 } \\
\text { Cofrentes } \\
\text { Dresden } 3 \\
\text { Grand Gulf } \\
\text { LaSalle } \\
\text { Santa Maria de Garona }\end{array}$ & $\begin{array}{r}\text { G11 } \\
\text { G8 } \\
\text { G10 } \\
\text { G10 } \\
\text { G5 } \\
\text { G12 } \\
\text { G9 } \\
\text { G3 }\end{array}$ & $\begin{array}{l}\text { TRAC-BF1 } \\
\text { TRAC-BD1 } \\
\text { TRAC-BF1 } \\
\text { TRAC-BF1 } \\
\text { TRAC-BD2 } \\
\text { TRAC-BD1 } \\
\text { TRAC-BF1 } \\
\text { TRAC-BF1 }\end{array}$ & $\begin{array}{l}\text { No } \\
\text { Yes } \\
\text { No } \\
\text { Yes } \\
\text { Yes } \\
\text { Yes } \\
\text { Yes } \\
\text { Yes }\end{array}$ & $\begin{array}{l}\text { INEL } \\
\text { INEL } \\
\text { INEL } \\
\text { CAMP } \\
\text { INEL } \\
\text { INEL } \\
\text { INEL } \\
\text { CAMP }\end{array}$ \\
\hline
\end{tabular}




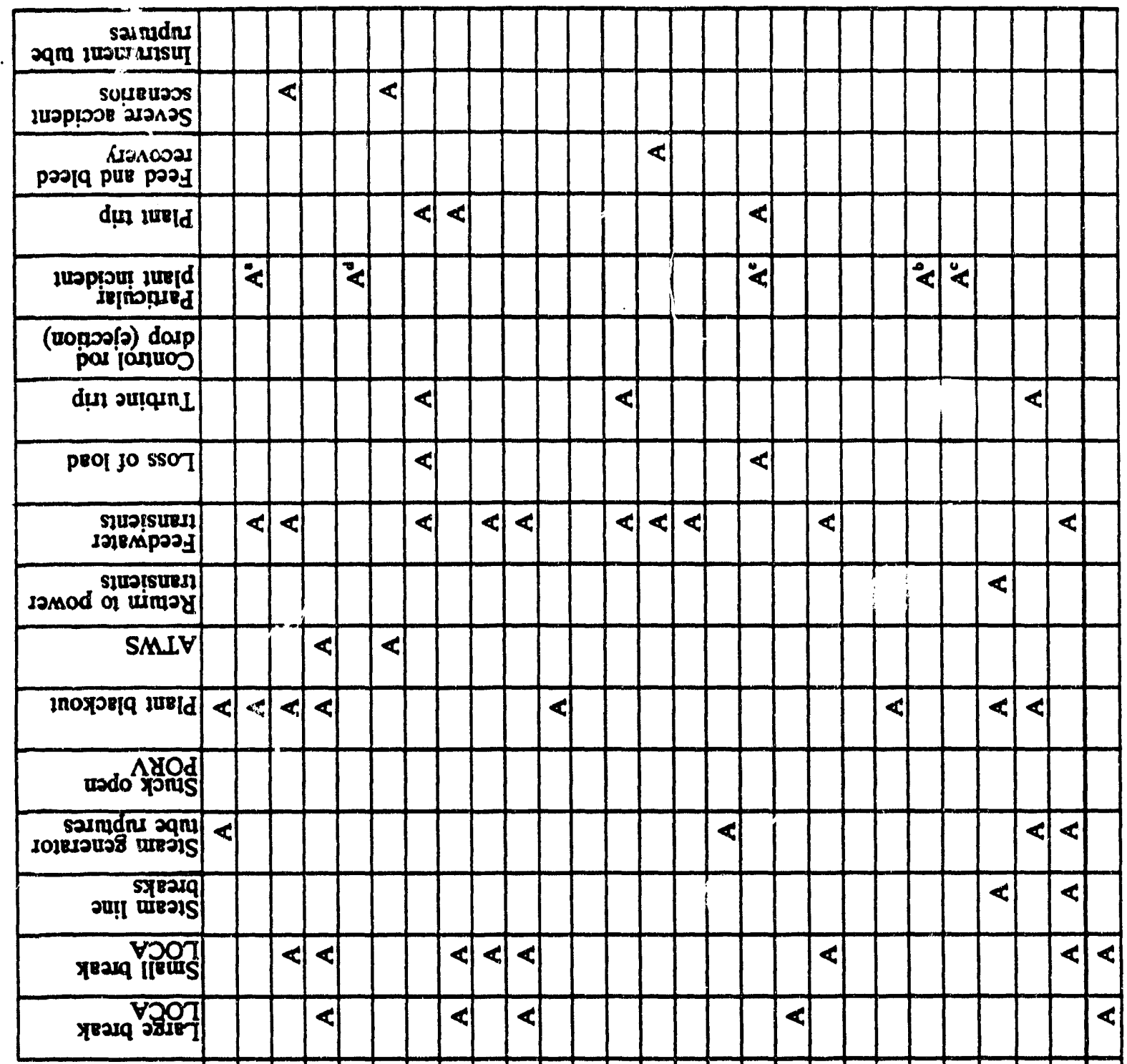

交
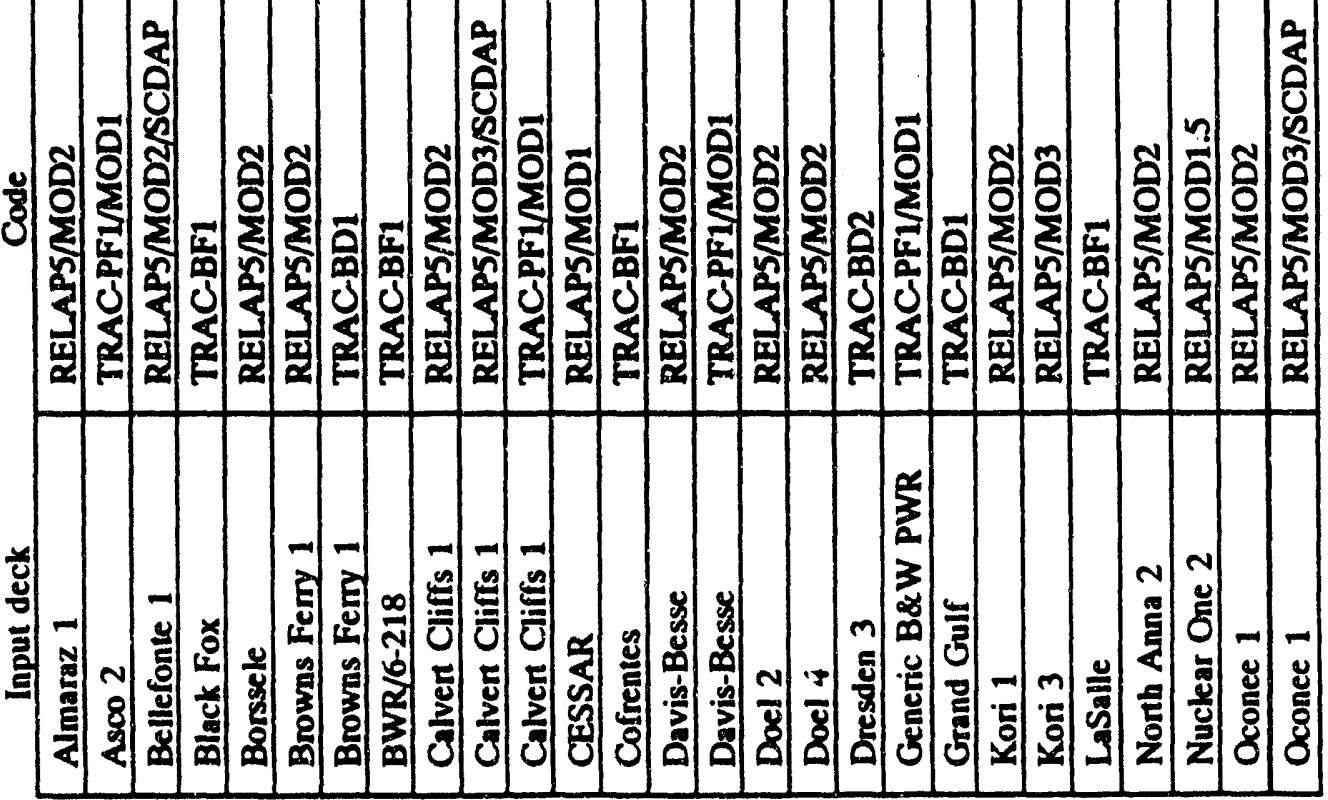


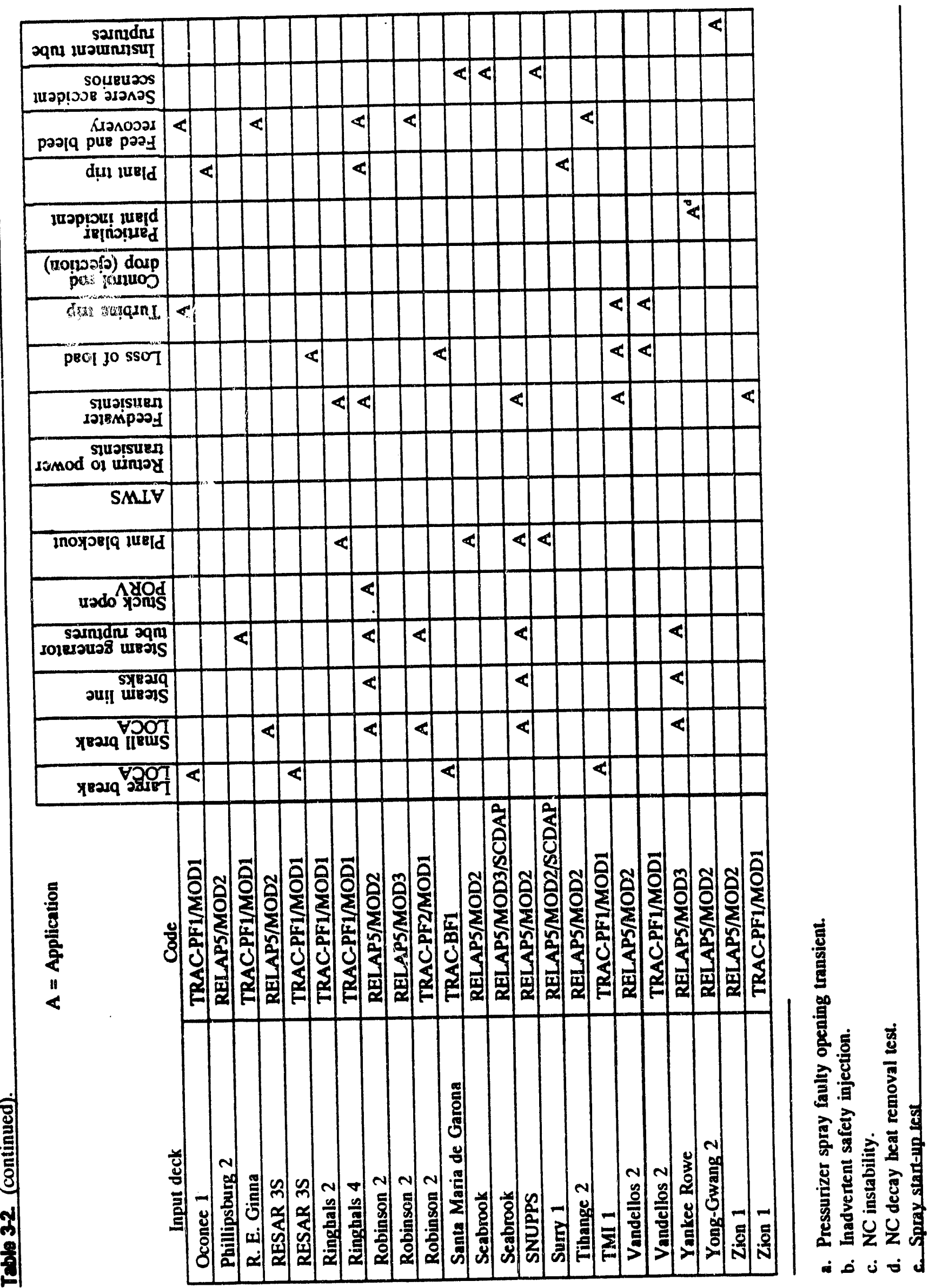




\section{PC BASED DATABASE}

The information gathered in this survey is most easily stored in a PC based database. As new information becomes available, it can be added to the database. Furthermore, information is easily accessed with a query program. Information on models for a particular plant may be queried; should there be none, the database will respond with information on models for similar plants. Models of plants belonging to a particular classification, models that run under a particular code, or models useful for a specific application may also be queried for and obtained.

Input decks may be queried by plant name, group, type, or vendor, by the code they run under, by application that the model may be used for, or by any combination of these specifications. The selection of these search parameters is menu driven. After selection of the parameters, a search is initiated which will locate the plant decks which most closely match the requirements. Therefore, if no decks exist for the specified plant, decks of plants from the same group, type, or vendor, in that order, will be located. The information may be viewed on the screen, or sent to a file or printer.

An input program allows for maintenance of the database.

The data collected for each NPP considered in the survey is discussed in Appendix A, and the data from input decks located in the survey is given.

Instructions for using the database are found in Appendix B.

The query and input programs may be run under dBASE III PLUS or FoxBASE+ on IBM or similar machines. A runtime module is included with the compiled codes for those users without access to either of the above mentioned database management systems. The database and programs may be acquired by sending one 5.25" floppy disk to

John Determan

MS 2403

P. O. Box 1.625

Idaho Falls, ID 83415 


\section{REFERENCES}

1. Correspondence-J. A. Dearien, to R. E. Tiller, Categorization of BWR and PWR Plants for SASA Work, JAD-220-80, August 28, 1980.

2. James Varley, World Nuclear Industry Handbook 1992, Nuclear Engineering International, Sutton England.

3. G. Gerth, Assessment Study of RELAPS/MOD2 Cycle 36.04 Based on the Commissioning Test Reactor Trip at Full Load at the Phillipsburg 2 Nuclear Power Plant, Office of Nuclear Regulatory Research, U.S. Nuclear Regulatory Commission, NUREG/IA-0057, April 1992.

4. A. Lopez, Application of Full Power Blackout for C.N. Almaraz with RELAP5/MOD2, Central Nuclear de Almaraz, ICAP-00205, ICSP-AL-BOUT-R, June 1991.

5. F. Reventos, Assessment and Application of Blackout Transients at Asco NPP with RELAP5/MOD2, Asociacion Nuclear Asco, ICAP-00201, ICSP-AS-BOUT-R, June 1991.

6. P. Moeyaert, et al, Assessment Study of RELAP5/MOD2, Cycle 36.04, Based on Spray Start-up test for DOEL-4, Office of Nuclear Regulatory Research, U.S. Nuclear Regulatory Commission, NUREG/IA-0020, July 1989.

7. Kyun-Tae Kim, et al, Assessment of RELAP5/MOD3 Versio\%w5m5 Using Inadvertent Safety Injection Data of Kori $U$. it 3 Plant, Korea Institute of Nuclear Safety, ICAP-00213.

8. A. Sjoberg, Assessment of TRAC-PF1/MOD1 Against an Inadvertent Feedwater Line Isolation Transient in the Ringhals 4 Power Plant, Office of Nuclear Regulatory Research, U.S. Nuclear Regulatory Commission, NUREG/IA-0038, March 1992.

9. G.P. Rouel, et al, Assessment Study of RELAP5/MOD2 Cycle 36.05 Based on the Tihange-2 Reactor Trip of January 11, 1983, Office of Nuclear Regulatory Research, U.S. Nuclear Regulatory Commission, NUREG/IA-0044, March 1992.

10. A. Querol, et al, Assessment of TRAC-PF1/MOD1 Against a Load Rejection from $100 \%$ to $50 \%$ in the Vandellos II Nuclear Power Plant, Union Iberoamericana de Tecnologia Electrica, ICAP00175, ICSP-V2R50-T, October 1990.

11. N. Arne, et al, Assessment of RELAP5/MOD2 Computer Code Against the Natural Circulation Test Data from Yong-Gwang Unit 2, Korea Electric Power Corporation, ICAP-00208.

12. F. Pelayo, et al, Assessment of TRAC-PF1/MOD1 Against an Inadvertent Steam Line Isolation Valve Closure in the Ringhals 2 Power Plant, Studsvik Energiteknik A.B., ICAP-00143, Studsvik/NP-88/14, February 1988. 
13. L. Winters, Assessment of RELAPS/MOD2 Against a Natural Circulation Experiment in Nuclear Power Plant Borssele, Netherlands Energy Research Foundation, ICAP-00136, ECN-89-91, November 1989.

14. E.J. Stubbe, Assessment Study of RELAP5/MOD2 Cycle 36.01: Based on the Doel 2 Steam Generator Tube Rupture Incident of June 1979, Office of Nuclear Regulatory Research, U.S. Nuclear Regulatory Commission, NUREG/IA-0008, October 1986.

15. B. Chung, et al, Assessment of RELAPS/MOD2 Code Using Loss of Offsite Power Transient Data of KNU \#1 Plant, Office of Nuclear Regulatory Research, U.S. Nuclear Regulatory Commission, NUREG/LA-0030, April 1990.

16. J.V. Lopez, et al, Recirculation Suction Large Break LOCA Analysis of Santa Maria de Garona Nuclear Power Plant Using TRAC-BF1 (G1J1), Polytechnical University of Madrid, ICAP-00188, ICSP-GA-LOCA-T, February 1991.

17. F. Castrillo, et al, Assessment of the "Turbine Trip Transient" in Cofrentes NPP with TRAC-BF1, Hidroelectrica Espanola, ICAP00202, ICSP-CO-TTRIP-T, June 1991. 
Appendix A

Data Gathered in the Survey

A-1 


\section{Appendix A}

\section{Data Gathered in the Survey}

The data can be divided into three categories: NPP data, input deck data, and report data. For all domestic NPPs and foreign plants similar to domestic plants for which thermal-hydraulic input decks are known to exist the information which categorizes them, specifically, vendor, type and similarity grouping is included in the database. It is this information that allows the query program to determine what other plant models could be of use when no model information is available for the plant in question. Other helpful information includes the plant's rated power output, the number and geometry of fuel assemblies in the reactor, the plant's location and licensing date.

Information regarding the status and capabilities of each plant deck considered in the survey has been gathered. The decks are identified by the plant name and theimal-hydraulic code related to each deck. The QA status and availability of each deck are noted, as are references to it. Documented assessments and applications of each deck are listed. The steady state conditions available are listed. A brief model description is included.

The custodian organization of each deck is noted, and telephone numbers of administrative and technical contacts are given. The technical contact is important because the information about each deck must be considered as representative, rather than as exhaustive; further information may be obtained by consulting the technical contact.

The report number, issue date, title, and principle author for every reference are included in the database.

Below is the database output for the decks located in the survey. 
PLANT:

Location:

Vendor:

License Date:

Utility:

Type:

MWt:

MWe:

\# of Bundles:

Group \#:

Code:

Q/A:

Organization:

Admin. Contact:

Technical Contact:

\section{REFERENCES}

Report Number:

Issue Date:

Author:

Title:

Report Number:

Issue Date:

Author:

Title:

Steady States:

Assessments:

Applications:

COMPONENT DESCRIPTION

Core:

Vessel:

Containment:

Balance of Plant:

Primary or Recirc:

Main Steam:

Control Systems:

Auxiliary Systems:

Fuel Rod Behavior:

Kinetics:
Almaraz 1

Spain

W

10/81

Central Nuclear de Almaraz

3-Loop

2696

930

$157 \quad 17 \times 17$

W6

RELAP5/MOD2

Y

EG\&G Idaho, Inc.

R R Schultz 208-526-9508

R R Schultz 208-526-9508

ICAP-00205

6/91

A. Lopez

APPLICATION OF FULL POWER BLACKOUT FOR

C.N. ALMARAZ WITH RELAP5/MOD2

ICAP-00220

9/91

A. Lopez

APPLICATION OF FULL POWER STEAM

GENERATOR TUBE RUPTURE EVENT FOR C. N.

ALMARAZ WITH RELAP5/MOD2

$100 \%$ power

Plant Blackout

Steam Generator Tube Ruptures

None reported

5 node core with average and hot channels modeled

No guide tube flow modeled

Pressure boundary

No feedtrain, detailed SGs

One double loop, one single loop

Detailed steam lines

Detailed trip and control system

ECCS, AFW

None

Power input table only 
PLANT:

Location:

Vendor:

License Date:

Utility:

Type:

MWt:

MWe:

\# of Bundles:

Group \#:

Code:

Q/A:

Organization:

Admin. Contact:

Technical Contact:

\section{REFERENCES}

Report Number:

Issue Date:

Author:

Title:

Report Number:

Issue Date:

Author:

Title:

Report Number:

Issue Date:

Author:

Title:

Steady States:

Assessments:

Applications:
Asco 2

Spain

W

2/86

Asociacion Nuclear Asco

3-Loop

2696

930

$157 \quad 17 \times 17$

W6

TRAC-PF1/MOD1

Y

EG\&G Idaho, Inc.

R R Schultz 208-526-9508

R R Schultz 208-526-9508

ICAP-00217

$6 / 91$

R. Martinez, et al

ASSESSMENT OF TRAC-PF1/MOD1 AGAINST A LOSS OF MAIN FEEDWATER IN ASCO NUCLEAR POWER PLANT

ICAP-00201

$6 / 91$

F. Reventos ASSESSMENT AND APPLICATION OF BLACKOUT TRANSIENTS AT ASCO NPP WITH RELAP5/MOD2

ICAP-00203

$6 / 91$

F. Reventos

ASSESSMENT OF A PRESSURIZER SPRAY FAULTY OPENING TRANSIENT AT ASCO NPP WITH RELAP5/MOD2

$100 \%$ power

Feedwater Transients

Plant Blackout

None reported 
Core:

Vessel:

Containment:

Balance of Plant:

Primary or Recirc:

Main Steam:

Control Systems:

Auxiliary Systems:

Fuel Rod Behavior:

Kinetics:
1D, six axial levels

Simple vessel model

Pressure Boundary

No feedtrain, detailed SGs

One double loop, one single loop

detailed steam lines

SG level, turbine runback, steam dump,

PZR level, RCS temperature

ECCS modeled

None

Point kinetics 
PLANT:

Location:

Vendor:

License Date:

Utility:

Type:

MWt:

MWe:

\# of Bundles:

Group \#:

Code:

Q/A:

Organization:

Admin. Contact:

Technical Contact:

\section{REFERENCES}

Report Number:

Issue Date:

Author:

Title:

Report Number:

Issue Date:

Author:

Title:

Steady States:

Assessments:

Applications:
Bellefonte 1

Scottsboro, Ala.

B\&W

indefinite

Tennessee Valley Authority

2-Loop High

3640

1213

$20517 \times 17$

B1

RELAP5/SCDAP/MOD2

Y

EG\&G Idaho, Inc.

C A Dobbe 208-526-9424

C A Dobbe 208-526-9424

EGG-NTP-6704

$10 / 84$

C. A. Dobbe, et. al.

ANALYSIS OF A STATION BLACKOUT TRANSIENT

FOR THE BELLEFONTE PRESSURIZED WATER

REACTOR

NUREG/CR-4741

3/87

P. D. Bayless, et. al. FEEDWATER TRANSIENT AND SMALL BREAK LOSS OF COOLANT ACCIDENT ANALYSES FOR THE BELLEFONTE NUCLEAR PLANT

$100 \%$ Power/ $100 \%$ Flow

None

Small Break LOCA

Plant Blackout

Feedwater Transients

Severe Accident Scenarios

COMPONENT DESCRIPTION

Core:

Single channel and three channel core models were developed

Vessel:

2 node low's plenum, 13 node downcomer, 8 node upper plenum/ upperhead

Containment: 
Balance of Plant:

Primary or Recirc:

Main Steam:

Control Systems:

Auxiliary Systems:

Fuel Rod Behavior:

Kinetics:
Feedwater is included

Both loops are modeled in detail

Both steam lines are modeled in detail

SG level

Auxiliary feedwater, LPI, HPI, and accumulator are included

SCDAP input included

Power input table used 


$\begin{array}{ll}\text { PLANT: } & \text { Black Fox } \\ \text { Location: } & \text { N/A } \\ \text { Vendor: } & \text { GE } \\ \text { License Date: } & \text { N/A } \\ \text { Utility: } & \text { N/A } \\ \text { Type: } & \text { BWR/6 238" CONTAINMENT } \\ \text { MWt: } & 3579 \\ \text { MWe: } & 1205 \\ \text { \# of Bundles: } & 732 \quad 8 \times 8 \\ \text { Group \#: } & \text { G11 } \\ \text { Code: } & \text { TRAC-BF1/MOD } \\ \text { Q/A: } & \text { Y }\end{array}$

Organization: EG\&G Idaho, Inc.

Admin. Contact: $\quad$ G W Johnsen 208-526-9854

Technical Contact: R P Martin 208-526-2880

\section{REFERENCES}

Report Number: NUREG/CR-5882

Issue Date: $\quad 6 / 92$

Author:

R. P. Martin

Title:

TRAC-B Thermal-Hydraulic Analysis of the Black Fox Boiling Water Reactor

Steady States: $\quad$ 100\% Power; $40 \%$ Power/60\% Flow

Assessments: None

Applications: Large Break LOCA

Small Break LOCA

Plant Blackout

ATWS

\section{COMPONENT DESCRIPTION}

Core: $\quad$ Three CHAN components, twelve nodes each

Vessel: $\quad$ Eleven Axial levels, three radial rings

Containment: Pressure boundary

Balance of Plant: From last feedwater heater to turbine bypass

Priniary or Recirc: Two Recirculation loops modeled

Main Steam: $\quad$ MSIV, TBV, and TSV modeled

Control Systems: Recirc. pump speed, ECCS controls, Isolation controls

Alixilliary Systems: ECCS

Fuel Rod Behavior: None

Kinetics: Point kinetics 
PLANT:

Location:

Vendor:

License Date:

Utility:

Type:

MWt:

MWe:

\# of Bundles:

Group \#:

Code:

Q/A:

Organization:

Admin. Contact:

Technical Contact:

\section{REFERENCES}

Report Number:

Issue Date:

Author:

Title:

Steady States:

Assessments:

Applications:

COMPONENT DESC...PTION

Core:

Vessel:

Containment:

Balance of Plant:

Primary or Recirc:

Main Steam:

Control Systems:

Auxilliary Systems:

Fuel Rod Behavior:

Kinetics:
Borssele

Netherlands

KWU

$10 / 73$

NV Provinciale Zeeuwse Energie Maatschappij

2-Loop

1365

452

$121 \quad 15 \times 15$

W9

RELAP5/MOD2

$\mathbf{Y}$

EG\&G Idaho, Inc.

G W Johnsen 208-526-9854

R R Schultz 208-526-9508

ICAP-00136

$11 / 89$

L. Winters

Assessment of RELAPS/MOD2 Against a

Natural Circulation Experiment in

Nuclear Power Plant Borssele

$13 \mathrm{MWh}, 15.32 \mathrm{MPa}, 548.4 \mathrm{~K}$

Natural Circulation Experiment

None reported

Single channel, six level core core bypass, upper head leakage Pressure boundary Detailed SG, no feedtrain Two explicit loops, detailed pressurizer Detailed steamlines pressurizer pressure and level, SG level ECCs, volume control system None

None 
PLANT:

Location:

Vendor:

License Date:

Utility:

Type:

MWt:

MWe:

\# of Bundles:

Group \#:

Code:

Q/A:

Organization:

Admin. Contact:

Technical Contact:

\section{REFERENCES}

Report Number:

Issue Date:

Author:

Title:

Report Number: Issue Date:

Author:

Title:

Report Numùr:

Issue Date:

Author:

Title:

Report Number: Issue Date:

Author:

Title:
Browns Ferry 1

Decatur, Ala.

GE

$8 / 74$

Tennessee Valley Authority

BWR/4 251" ID CONTAINMENT 1

3293

1065

$7648 \times 8$

G8

RELAP5/MOD2

$\mathrm{Y}$

EG\&G Idaho, Inc.

G W Johnsen 208-526-9854

R R Schultz 208-526-9508

EGG-NTAP-5993

$8 / 82$

W. C. Jouse, et. al.

A RELAP5 ANALYSIS OF A BREAK IN THE SCRAM DISCHARGE VOLUME AT THE BROWNS FERRY UNIT ONE PLANT

EGG-SAAM-6397

$11 / 83$

W. C. Jouse

DRAFT PRELIMINARY REPORT (CODE) FOR

COMMENT RELAP5/MOD1.6 BWR/4 ATWS

DEMONSTRATION CALCULATION ON THE BROWNS

FERRY UNIT 1 NUCLEAR PLANT

EGG-NTAP-6002

9/82

R. R. Schultz, et. al.

THE STATION BLACKOUT TRANSIENT AT THE

BROWNS FERRY UNIT ONE PLANT A SEVERE

ACCIDENT SEQUENCE ANALYSIS

NUREG/CR-4165

$5 / 87$

R. J. Dallman

SEVERE ACCIDENT SEQUENCE ANALYSIS

PROGRAM - ANTICIPATED TRANSIENT WITHOUT

SCRAM SIMULATIONS FOR BROWNS FERRY 


\section{NUCLEAR PLANT UNIT 1}

Report Number:

Issue Date:

Author:

Title:

Steady States:

Assessments:

Applications:

\section{COMPONENT DESCRIPTION}

Core:

Vessel:

Containment:

Balance of Plant:

Primary or Recirc:

Main Steam:

Control Systems:

Auxiliary Systems:

Fuel Rod

Behavior:

Kinetics:
FIN NO. A6477

$7 / 84$

R. G. Hanson, et. al.

EFFECTS OF CONTROL SYSTEM FAILURES ON

TRANSIENTS AND ACCIDENTS AT A GENERAL

ELECTRIC BOILING WATER REACTOR

APPENDICES A. B. C. AND D

100\% Power $/ 100 \%$ Flow

None

ATWS

Severe Accident Scenarios

Average assembly and hot channel modeled 12 node assembly, 3 node bypass 4 node lower plenum, 5 node downcomer, 5 node upper plenum and steam dome Containment model available: CONTEMPT/ LT-028 model

Feedwater and turbines are modeled The two recirculation loops are lumped as one. The jet pumps are modeled.

Six node steam line. MSIV, SRVs and bypass valves are modelled Recirc. pump speed, FW, drain, FW heater extraction steam flow $\mathrm{HPCl}, \mathrm{LPCI}, \mathrm{RCIC}$, Control rod drive

FRAP-T6 modelling is used Point kinetics 
PLANT:

Location:

Vendor:

License Date:

Utility:

Type:

MWt:

MWe:

\# of Bundles:

Group \#:

Code:

Q/A:

Organization:

Admin. Contact:

Technical Contact:

\section{REFERENCES}

Report Number:

Issue Date:

Author:

Title:

Steady States:

Assessments:

Applications:

COMPONENT DESCRIPTION

Core:

Vessel:

Containment:

Balance of Plant:

Primary or Recirc:

Main Steam:

Control Systems:

Auxiliary Systems:

Fuel Rod Behavior:

Kinetics:
Browns Ferry 1

Decatur, Ala.

GE

$8 / 74$

Tennessee Valley Authority

BWR/4 251" ID CONTAINMENT 1

3293

1065

$7648 \times 8$

G8

TRAC-BD1/MOD1

$\mathrm{N}$

EG\&G Idaho, Inc.

G W Johnsen

208-526-9854

J D Burtt

208-526-9089

FIN NO. A6047

$11 / 84$

J. D. Burtt

DRAFT PRELIMINARY REPORT FOR COMMENT

TRAC-BD1/MOD1 ASSESSMENT USING BROWNS

FERRY BWR/4 STARTUP TEST DATA

$100 \%$ Power $/ 100 \%$ Flow

Feedwater Transients

Loss of Load

Turbine Trip

Plant Trip

None

1-dimensional 3 channel core. Average peripheral, and hot assemblies modeled 3-D vessel with 10 axial, and four radial regions

Pressure boundary

Feedwater modeled

Both loops are modeled with 3 nodes per loop. Jet pumps are modeled

2 node steam line with the MSIV, SRVs, bypass valve and the PRVs modeled

Recirculation pumps

None

None

Point kinetics 
PLANT:

Location:

Vendor:

License Date:

Utility:

Type:

MWt:

MWe:

\# of Bundles:

Group \#:

Code:

Q/A:

Organization:

Admin. Contact:

Technical Contact:

\section{REFERENCES}

Report Number:

Issue Date:

Author:

Title:

Report Number:

Issue Date:

Author:

Title:

Steady States:

Assessments:

Applications:
BWR/6-218

N/A

GE

N/A

N/A

BWR/6 218" CONTAINMENT

2894

N/A

$6248 \times 8$

G10

TRAC-BF1/MOD

$\mathrm{N}$

EG\&G Idaho, Inc.

P D Wheatley 208-526-9348

P D Wheatley 208-526-9348

EGG-NTAP-6073

$10 / 82$

P. D. Wheatley

Scaling Analysis of the FIST Facility

Using TRAC-BD1

NUREG/CR-4391

8/86

W. L. Weaver, et al

TRAC-BF1 Manual: Extensions to

TRAC-BD1/MOD1

$100 \%$ Power/100\% Flow

None

Plant Trip

Large Break LOCA

Small Break LOCA

\section{COMPONENT DESCRIPTION}

Core:

Three radial rings, one azimuthal sector two axial levels

Vessel:

Containment:

Four radial rings, one azimuthal sector nine axial levels

Pressure boundary

No feedtrain modelled

Two recirc loops modelled

Primary or Recirc:

Main Steam:

ADS, SRV modelled

Control Systems:

Steam line valve area

Auxilliary Systems:

HPCS, LPCS, and LPCI

None

Decay heat curves used 
PLANT:

Location:

Vendor:

License Date:

Utility:

Type:

MWt:

MWe:

\# of Bundles:

Group \#:

Code:

Q/A:

Organization:

Admin. Contact:

Technical Contact:

\section{REFERENCES}

Report Number:

Issue Date:

Author:

Title:

Steady States:

Assessments:

Applications:
Calvert Cliffs 1

Lusby, Md.

C-E

5/75

Baltimore Gas \& Electric Co.

2X4 Loop

2570

825

$217 \quad 14 \times 14$

C5

RELAP5/MOD2

Y

EG\&G Idaho, Inc.

G W Johnsen

208-526-9854

C B Davis
NUREG/CR-4966 V1

10/87

D. J. Hanson, et. al.

EVALUATION OF OPERATIONAL SAFETY AT

BABCOCK AND WILCOX PLANTS. VOLUME 1 -RESULTS OVERVIEW

$100 \%$ Power/ $100 \%$ Flow

None

Small Break LOCA

Feedwater Transients

COMPONENT DESCRIPTION

Core:

Vessel:

Containment:

Balance of Plant:

Primary or Recirc:

Main Steam:

Control Systems:

Auxiliary Systems:

Fuel Rod Behavior:

Kinetics:
3 Node core, no bypass modeled 2 node lower plenum, 7 node downcomer, 7 node upper plenum/ upper head 1 node containment Main feedwater and turbine are modeled All loops are modeled in detail Both steam lines modeled in detail Reactor coolant pressure regulator, pressurizer level, feedwater regulator Auxiliary feedwater, HPI, LPI, Accumulator, and charging systems

None

Input power table only 
PLANT:

Location:

Vendor:

License Date:

Utility:

Type:

MWt:

MWe:

\# of Bundles:

Group \#:

Code:

Q/A:

Organization:

Admin. Contact:

Technical Contact:

\section{REFERENCES}

Steady States:

Assessments:

Applications:
Calvert Cliffs 1

Lusby, Md.

C-E

5/75

Baltimore Gas \& Electric Co.

2X4 Loop

2570

825

$217 \quad 14 \times 14$

C5

RELAP5/SCDAP/MOD3

Y

EG\&G Idaho, Inc.

G W Johnsen 208-526-9854

J C Determan 208-526-2888

None

100\% Power/ 100\% Flow

None

Large Break LOCA

Small Break LOCA

Feedwater Transients

\section{COMPONENT DESCRIPTION}

Core:

Vessel:

Containment:

Balance of Plant:

Primary or Recirc:

Main Steam:

Control Systems:

Auxiliary Systems:

Fuel Rod Behavior:

Kinetics:
3 channel, 10 Node core, bypass modeled Split downcomer 1 node containment

Main feedwater and turbine are modeled All loops are modeled in detail Both steam lines modeled in detail Reactor coolant pressure regulator, pressurizer level, feedwater regulator Auxiliary feedwater, HPI, LPI, Accumulator, and charging systems SCDAP input for outer, middle, and center channels of core Input power table only 
PLANT:

Location:

Vendor:

License Date:

Utility:

Type:

MWt:

MWe:

\# of Bundles:

Group \#:

Code:

Q/A:

Organization:

Admin. Contact:

Technical Contact:

REFERENCES

Report Number:

Issue Date:

Author:

Title:

Report Number:

Issue Date:

Author:

Title:

Report Number: Issue Date:

Author:

TITLE:

Report Number:

Issue Date:

Author:

Title:
Calvert Cliffs 1

Lusby, Md.

C-E

5/75

Baltimore Gas \& Electric Co.

2X4 Loop

2570

825

$217 \quad 14 \times 14$

C5

TRAC-PF1/MOD1

Y

Los Alamos National Laboratory

J F Lime 505-667-4097

J F Lime 505-667-4097

NUREG/CR-4109

$2 / 85$

G. D. Spriggs, et. al.

TRAC-PF1 ANALYSES OF POTENTIAL PRESSURIZED-THERMAL-SHOCK TRANSIENTS AT CALVERT CLIFFS / UNIT 1 A COMBUSTION ENGINEERING PWR

LA-UR-85-2868

$8 / 85$

Rick Jenks

COOLDOWN TO RESIDENT-HEAT REMOVAL ENTRY

CONDENSATION USING ATMOSPHERIC DUMP

VALVES AND AUXILIARY PRESSURIZER SPRAY FOLLOWING A LOSS-OF-OFFSITE-POWER AT CC1

LA-UR-85-2039

1985

B. E. BOYACK

ATMOSPHERIC STEAM DUMP COOLDOWN OF CALVERT CLIFFS-1 FOLLOWING A SMALL-BREAK LOSS-OF-COOLANT ACCIDENT WITH FAILURE OF THE HIGH PRESSURE INJECTION SYSTEM

LA-UR-85-0960

1985

R. Jenks

ALTERNATIVE COOLDOWN METHODS FOLLOWING A 
LOSS-OF-OFFSITE POWER (LOSP) EVENT AT

CALVERT CLIFFS UNIT 1

Report Number:

Issue Date:

Author:

Title:

Report Number: Issue Date:

Author:

Title:

Report Number: Issue Date:

Author:

Title:

Report Number: Issue Date:

Author:

Title:

Report Number: Issue Date:

Author:

Title:

Report Number: Issue Date:

Author:

Title:
LA-UR-85-0424

1985

R. Jenks

COOLDOWN PERFORMANCE CAPABILITY FOR

CALVERT CLIFFS-1 ASSUMING VARIOUS

ATMOSPHERIC DUMP VALVE SIZES

LA-UR-84-3947

1984

R. P. Jenks

COOLDOWN TO RESIDUAL HEAT REMOVAL ENTRY CONDITIONS USING ATMOSPHERIC DUMP VALVES

AND AUXILIARY PRESSURIZER SPRAY

FOLLOWING A LOSS-OF-OFFSITE POWER AT

CALVERT CLIFFS-UNIT 1

LA-UR-84-3860

1984

B. Boyack

Use of an Atmospheric Steam Dump

PROCEDURE TO COOL AND DEPRESSURIZE

CALVERT CLIFFS-1 FOLLOWING A SMALL-BREAK LOSS-OF-COOLANT ACCIDENT WITH FAILURE OF THE HIGH-PRESSURE INJECTION SYSTEM

LA-UR-84-3589

1984

R. P. Jenks

COOLDOWN PERFORMANCE CA

CALVERT CLIFFS-1 ASSUMING VARIOUS

ATMOSPHERIC DUMP VALVE SIZES

LA-UR-84-3266

1984

E. Horley

COMBINED MAIN-STEAM-LINE BREAK / LOSS-OF-FEEDWATER TRANSIENT IN THE

CALVERT CLIFFS- 1 PWR

LA-UR-84-3006

1984

R. Jenks

ALTERNATIVE COOLDOWN METHODS FOLLOWING A LOSS-OF-OFFSITE POWER (LOSP) EVENT AT 


\section{CALVERT CLIFFS UNIT 1}

Report Number: Issue Date:

Author:

Title:

Report Number: Issue Date:

Author:

Title:

Report Number: Issue Date:

Author:

Title:

Report Number: Issue Date:

Author:

Title:

Report Number: Issue Date:

Author:

Title:

Report Number: Issue Date: Author:

Title:

Report Number: Issue Date:

Author:

Title:
LA-UR-84-1973

1984

B. E. Boyack, et al AN ASSESSMENT OF THI UTILITY OF FEED-

AND-BLEED OPERATING A APS (CALVERT CLIFFS-1)

LA-UR-83-3375

1983

R. Smith

LOSS-OF-FEEDWATER TRANSIENT FOR THE

CALVERT CLIFFS-1 PWR

LA-UR-83-3260

1983

Russel Smith

LOSS-OF-OFFSITE-POWER TRANSIENT FOR THE

CALVERT CLIFFS-1 PWR

LA-UR-83-2895

1983

J. Lime

REVIEW OF THE TRAC-PF1 CALVERT CLIFFS-1

PLANT MODEL

LA-UR-85-4146

1985

B. E. Boyack

AN ALTERNATE METHOD OF DECAY-HEAT REMOVAL IN A C \& E PLANT FOLLOWING A SBLOCA

LA-UR-85-3501

9/85

G. Willcutt

TECHNICAL EVALUATION REPORT FOR THE TRAC ANALYSES OF SMALL-BREAK LOSS-OF-COOLAN'T ACCIDENTS TO EVALUATE COMBUSTION ENGINEERING MODELS USED TO ESTABLISH REACTOR-COOLANT-PUMP TRIP SET-POINT CRITERIA

LA-UR-85-2038

1985

B. E. Boyack

AN ALTERNATIVE METHOD OF DECAY-HEAT

REMOVAL IN COMBUSTION-ENGINEERING

PLANT FOLLOWING A SMALL-BREAK 


\section{LOSS-OF-COOLANT ACCIDENT}

Report Number: Issue Date:

Author:

Title:

Report Number: Issue Date:

Author:

Title:

Report Number: Issue Date:

Author:

Title:

Report Number: Issue Date:

Author:

Title:

Report Number: Issue Date:

Author:

Title:

Report Number: Issue Date:

Author:

Title:

Report Number:

Issue Date:

Author:
LA-UR-85-036

1985

B. E. Boyack

AN ALTERNATIVE METHOD OF DECAY-HEAT

REMOVAL IN A C-E PLANT FOLLOWING A

SBLOCA

LA-UR-84-2809

9/84

J. Kocnig, et al

TRAC-PF-1 ANALYSES OF POTENTIAL

PRESSURIZED-THERMAL-SHOCK TRANSIENTS AT

A COMBUSTION ENGINEERING PWR

LA-UR-84-2757

1984

J. Koenig, et al

TRAC/PF1 ANALYSES OF POTENTIAL

PRESSURIZED-THERMAL-SHOCK TRANSIENTS AT A COMBUSTION-ENGINEERING PWR

LA-UR-84-2083

$7 / 84$

G. Spriggs, et al

TRAC-PF1 ANALYSES OF POTENTIAL PRESSURIZED-THERMAL-SHOCK TRANSIENTS AT A COMBUSTION-ENGINEERING PWR

LA-UR-84-0502

1984

J. Koenig, et al

TRAC-PF1 ANALYSES OF POTENTIAL PRESSURIZED-THERMAL-SHOCK TRANSIENTS AT A COMBUSTION-ENGINEERING PWR

LA-UR-84-0131

1984

J. Koenig, et al

EFFECTS OF PUMP OPERATION DURING POSTULATED STEAM-LINE-BREAK IN A CE

PLANT

LA-UR-83-1913

1983

J. Koenig 
Title:

Report Number:

Issue Date:

Author:

Title:

Steady States:

Assessments:

Applications:

\section{COMPONENT DESCRIPTION}

Core:

Vessel:

Containment:

Balance of Plant:

Primary or Recirc:

Main Steam:

Control Systems:

Auxiliary Systems:

Fuel Rod Behavior:

Kinetics:
TRAC THERMAL-HYDRAULIC PREDICTIONS OF A STEAM-LINE-BREAK IN A CE PLANT ANS PAPER.

LA-UR-82-0914

$4 / 82$

J. F. Lime

TRAC MODELING OF A COLD-LEG SMALL-BREAK TRANSIENT IN A COMBUSTION ENGINEERING PRESSURIZED WATER REACTOR

$100 \%$ power

None

Plant Blackout

6 level, 1-D

1-Dimensional

Pressure boundary

Detailed secondary

All loops modeled

MSIVs, atmospheric dump, SRVs

MFP speed, SG level, PZR heater, steam

bypass dump, makeup/letdown

Auxiliary feedwater, HPI

Basic rod conduction

Input power table only 
PLANT:

Location:

Vendor:

License Date:

Utility:

Type:

MWt:

MWe:

\# of Bundles:

Group \#:

Code:

Q/A:

Organization:

Admin. Contact:

Technical Contact:

\section{REFERENCES}

Steady States:

Assessments:

Applications:

\section{COMPONENT DESCRIPTION}

Core:

Vessel:

Containment:

Balance of Plant:

Primary or Recirc:

Main Steam:

Control Systems:

Auxiliary Systems:

Fuel Rod Behavior:

Kinetics:
CESSAR

N/A

C-E

N/A

N/A

2x4 Loop

N/A

N/A

N/A

C5

RELAP5/MOD2

N

EG\&G Idaho, Inc.

P D Bayless 208-526-9027

P D Bayless 208-526-9027

None

$100 \%$ power

None

None reported

6-node, single channel core with hot channel

Includes core bypass and upper

head leak path

Pressure boundary

No feedtrain

Both loops explicit, detailed pressurizer

Includes MSIVs and stop valve

None

ECCS. makup, letdown, SG aux. feed

None

Point kinetics 
PLANT:

Location:

Vendor:

License Date:

Utility:

Type:

MWt:

MWe:

\# of Bundles:

Group \#:

Code:

Q/A:

Organization:

Admin. Contact:

Technical Contact:

\section{REFERENCES}

Report Number:

Issue Date:

Author:

Title:

Report Number:

Issue Date:

Author:

Title:

Steady States:

Assessments:

Applications:

\section{COMPONENT DESCRIPTION}

Core:

Vessel:

Containment:

Balance of Plant:

Primary or Recirc:

Main Steam:

Control Systems:

Auxiliary Systems:

Fuel Rod Behavior:

Kinetics:
Cofrentes

Spain

$\mathrm{GE}$

$3 / 85$

Hidroelectrica Espanola

BWR/6 232" ID CONTAINMENT

2894

958

$624 \quad 8 \times 8$

GiO

TRAC-BF1/MOD

Y

EG\&G Idaho, Inc.

$\begin{array}{ll}\text { R R Schultz } & 208-526-9508 \\ \text { R R Schultz } & 208-526-9508\end{array}$

ICAP־00202

$6 / 91$

F. Castrillo

ASSESSMENT OF THE "TURBINE TRIP

TRANSIENT" ${ }^{n}$ IN/ COFRENTES NPP WITH

TRAC-BF1

ICAP-00189

$2 / 91$

F. Castrillo

Assessment of the "One Feedwater Pump

Trip Transient" in Cofrentes NPP with

TRAC-BF1

71\% power,94\% flow; $95 \%$ power,94\% flow Turbine Trip

Feedwater Transients

None reported

3 radial rings, 2 axial levels

4 radial rings, 8 axial levels

Pressure boundary

No feedtrain

Both recirc. loops modeled, all jetpumps

on each line lumped together

Detailed steam line

Pressure control, feedwater control,

Recirc. flow control (manual mode)

LPCI, HPCI, LPCS

None

Point kinetics 


\section{PLANT:}

Location:

Vendor:

License Date:

Utility:

Type:

MWt:

MWe:

\# of Bundles:

Group \#:

Code:

Q/A:

Orga: vation:

Admin. Contact:

Technical Contact:

\section{REFERENCES}

Report Number:

Issue Date:

Author:

Title:

Steady Siates:

Assessments:

Applications:
Davis-Besse

Oak Harbor, Ohio

B\&W

7778

Toledo Edison Co.

2-Loop High

2633

874

$17715 \times 15$

B2

RELAP5/MOD2

Y

EG\&G Idaho, Inc.

G W Johnsen 208-526-9854

C B Davis 208-526-9470
NUREG/CR-4946

8/87

こ. B. Davis

DAVIS-BESSE UNCER? AINTY STUDY

92\% Power

None

Feedwater Transients

Feed and Bleed Pecovery

\section{COMPONENT DESCRIPTION}

Core:

Vessel:

Containment:

Balance of Plant:

Primary or Recirc:

Main Steam:

Control Systems:

Auxiliary Systems:

Fuel Rod Behavior:

Kinetics:
6 node core

2 node lower plenum, 7 node downcomer, 7 node upper plenum/upper head

Pressure boundary

Main feedwater is modeled

Both legs modeled

2 node steamline. MSIVs and condensers are modeled.

Steamline safety valves, makeup HPI, LPI

SG level, pressurizer liquid level

Makeup, HPI, LPI, condenser

None

Power vs. time table 
PLANT:

Location:

Vendor:

License Date:

Utility:

Type:

MWt:

MWe:

\# of Bundles:

Group \#:

Code:

Q/A:

Orga ization:

Admin. Contact:

Technical Contact:

\section{REFERENCES}

Report Number:

Issue Date:

Author:

Title:

Report Number: Issue Date:

Author:

Title:

Report Number:

Issue Date:

Author:

Title:

Steady States:

Assessments:

Applications:
Davis-Besse

Oak Harbor, Ohio

B\&W

$7 / 78$

Toledo Edison Co.

2-Loop High

2633

874

$177 \quad 15 \times 15$

B2

TRAC-PF1/MOD1

Y

Los Alamos National Laboratory

J F Lime 505-667-4097

J F Lime 505-667-4097

LA-UR-86-1782

1986

RAPID-RESPONSE ANALYSIS OF THE

DAVIS-BESSE LOSS-OF-FEEDWATER EVENT ON

JUNE 9, 1985

LA-UR-86-0205

$1 / 86$

J. F. Lime, et al

RAPID-RESPONSE ANALYSIS OF THE

DAVIS-BESSE LOSS-OF-FEEDWATER EVENT OF

JUNE 9, 1985

LA-UR-85-3083

$8 / 85$

J. F. Lime, et al

RAPID-RESPONSE ANALYSIS OF THE

DAVIS-BESSE LOSS-OF-FEEDWATER EVENT ON

JUNE 9. 1985

$100 \%$ core power

Feedwater Transients

Feedwater Transients 


\section{COMPONENT DESCRIPTION}

Core:

Vessel:

Containment:

Balance of Plant:

Primary or Recirc:

Main Steam:

Control Systems:

Auxiliary Systems:

Fuel Rod Behavior:

Kinetics:
1 radial ring, 2 azimuthal sectors,

4 axial levels

2 radial rings, 2 azimuthal sectors, 12 axial levels; guide tubes, vent valves

Pressure Boundary

Detailed Secondary

Both loops modeled in detail

Includes turbine stop valves

Complete SG control, PORV

Auxiliary feedwater

8 radial condensation nodes in fuel, gap and clad

Input power table only 
PLANT:

Location:

Vendor:

License Date:

Utility:

Type:

MWt:

MWe:

\# of Bundles:

Group \#:

Code:

Q/A:

Organization:

Admin. Contact:

Technical Contact:

\section{REFERENCES}

Report Number:

Issue Date:

Author:

Title:

Steady States:

Assessments:

Applications:

COMPONENT DESCRIPTION

Core:

Vessel:

Containment:

Balance of Plant:

Primary or Recirc:

Main Steam:

Auxilliary Systems:

Fuel Rod Behavior:

Kinetics:
Doel 2

Belgium

W

$12 / 75$

Indivision Doel

2-Loop

1187

392

$12114 \times 14$

W9

RELAP5/MOD2

$\mathbf{Y}$

EG\&G Idaho, Inc.

G W Johnsen 208-526-9854

R R Schultz 208-526-9508

NUREG/IA-0008

9/86

E.J. Strubbe

Assessment Study of RELAP5/MOD2 Cycle

36.01: Based on the DOEL-2 Steam

Generator Tube Rupture Incident of June 1979

$6 \mathrm{MWt}$ decay, 155 bar, $255 \mathrm{C}$

Steam Grnerator Tube Ruptures

None reported
Single channel, four node core, constant

decay heat

Core bypass and upper head leakage paths

modeled

Pressure Boundary

No feed train, detailed steam generators

Both loops explicit, detailed

pressurizer

Atmospheric dump valvesControl Systems: Basic trip system

AUX feed, HPSI, Makeup and letsown

None

None 
PLANT:

Location:

Vendor:

License Date:

Utility:

Type:

MWt:

MWe:

\# of Bundles:

Group \#:

Code:

Q/A:

Organization:

Admin. Contact:

Technical Contact:

\section{REFERENCES}

Report Number:

Issue Date:

Author:

Title:

Report Number:

Issue Date:

Author:

Title:

Report Number:

Issue Date:

Author:

Title:

Steady States:

Assessments:

Applications:
Doel 4

Belgium

W*

$7 / 85$

Societes Reunies d'Energie du Bassin de l'Escaut

3-Loop

3000

1010

$157 \quad 17 \times 17$

W6

RELAP5/MOD2

Y

EG\&G Idaho, Inc.

G W Johnsen 208-526-9854

R R Schultz 208-526-9508

NUREG/IA-0020

7/89

P. Moeyaert, et al Assessment Study of RELAP5/MOD2, Cycle 36.04 Based on Spray Start-up Test for

Doel-4

NUREG/IA-0043

3/92

E.J. Strubbe, et al

Assessment Study of RELAP5/MOD2 Cycle 36.04 Based on the Doel-4 Manual Loss of Load Test of November 23, 1985

NUREG/IA-0051

3/92

M. De Vlaminck, et al Assessment Study of RELAP5/MOD2 Cycle 36.05 Based on the Doel 4 Reactor Trip of November 22, 1985

$0 \%$ core power, 155 bars; $98 \%$ core power

Loss of Load

Plant Trip

None reported 


\section{COMPONENT DESCRIPTION}

Core:

Vessel:

Containment:

Balance of Plant:

Primary or Recirc:

Main Steam:

Control Systems:

Auxilliary Systems:

Fuel Rod Behavior:

Kinetics:
Single channel, five level core

Core bypass and upper head leakage

Pressure boundary

Detailed SG, no feedtrain

All three loops explicit

Detailed Steamlines

Pressurizer pressure, feedwater, steamdump

SI, accumulators, chemical and volume

None

Point kinetics 
PLANT:

Location:

Vendor:

License Date:

Utility:

Type:

MWt:

MWe:

\# of Bundles:

Group \#:

Code:

Q/A:

Organization:

Admin. Contact:

Technical Contact:

\section{REFERENCES}

Report Number:

Issue Date:

Author:

Title:

Steady States:

Assessments:

Applications:

COMPONENT DESCRIPTION

Core:

Vessel:

Containment:

Balance of Plant:

Primary or Recirc:

Main Steam:

Control Systems:

Auxiliary Systems:

Fuel Rod Behavior:

Kinetics:
Dresden 3

Morris, Ill.

GE

$11 / 71$

Commonwealth Edison Co.

BWR/3 251" ID CONTAINMENT 1

2527

773

$7249 \times 9$

G5

TRAC-BD1/MOD

$\mathrm{N}$

EG\&G Idaho, Inc.

G W Johnsen 208-526-9854

C B Davis $\quad 208-526-9470$

EGG-NTAP-6090

9/82

C. B. Davis

AN ANALYSIS OF A SPECTRUM OF LARGE-BREAK LOCAS IN A BWR/3 USING TRAC-BD1

$102 \%$ rated power

None

Large Break LOCA

10 cell core

13 axial nodes, 4 radial, 1 azimuthal

Pressure boundary

Main feedwater is modeled

"Intact" and "broken" loops are modeled.

There are 14 nodes per loop with the

2 node steam line with the MSIV included

Feedwater steam line flow and pressure

(may not be functional)

HPCI, LPCS, ADS

None

Point kinetics 
PLANT:

Location:

Vendor:

License Date:

Utility:

Type:

MWt:

MWe:

\# of Bundles:

Group \#:

Code:

Q/A:

Organization:

Admin. Contact:

Technical Contact:

\section{REFERENCES}

Report Number:

Issue Date:

Author:

Title:

Report Number:

Issue Date:

Author:

Title:

Report Number:

Issue Date:

Author:

Title:

Report Number:

Issue Date:

Author:

Title:
Generic B\&W PWR

N/A

B\&W

N/A

N/A

2-Loop Low

N/A

N/A

$17715 \times 15$

B3

TRAC-PF1/MOD1

Y

Los Alamos National Laboratory

M W Cappiello 505-667-4097

M W Cappiello 505-667-4097

LA-UR-83-3079

$4 / 86$

C. E. Watson

A TRAC-PF1 CALCULATION OF A

TURBINE-BYPASS-VALVE FAILURE ACCIDENT IN

A GENERIC B \& W PLANT

LA-UR-83-3001

1983

R.J. Henninger, et al

LOSS-OF-OFFSITE-POWER-INITIATED

LOSS-OF-FEEDWATER TRANSIENTS FOR B \& W

PLANTS

LA-UR-83-2786

1983

R. J. Henninger, et al

SMALL-BREAK LOCA RECOVERY IN B \& W PLANTS

LA-UR-83-2559

1983

C. Watson

MODEL CHANGES, STEADY-STATE CONDITIONS AND TRANSIENT ASSUMPTIONS FOR A TRAC CALCULATION OF A TURBINE-BYPASS VALVE

FAILURE IN A GENERIC B \& W PLANT 
Report Number:

Issue Date:

Author:

Title:

Report Number:

Issue Date:

Author:

Title:

Report Number:

Issue Date:

Author:

Title:

Report Number:

Issue Date:

Author:

Title:

Report Number:

Issue Date:

Author:

Title:

Steady States:

Assessments:

Applications:
LA-UR-83-0818

1983

J. R. Ireland

SMALL-BREAK LOCA RECOVERY IN B \& W

PLANTS

LA-UR-83-0657

10/83

B. E. Boyack, et al TRAC-PF1 CALCULATION OF B \& W PRESSURIZED THERMAL SHOCK

TRANSIENTS-OVERCOOLING CAUSED BY TURBINE BYPASS VALVE FAILURES

LA-UR-82-3676

1982

B. E. Boyack, et al

TRAC-PF1 CALCULATION OF B \& W

PRESSURIZED THERMAL SHOCK

TRANSIENTS-OVERCOOLING CAUSED BY TURBINE BYPASS VALVE FAILURES

LA-UR-82-2841

1982

B. Bassett

CALCULATIONS OF PRESSURIZED THERMAL SHOCK TRANSIENTS IN B \& W PLANTS

LA-UR-82-0911

$4 / 82$

B. Bassett

TRAC ANALYSIS OF STEAM-GENERATOR

OVERFILL TRANSIENTS FOR

BABCOCK-AND-WILCOX PRESSURIZED WATER

REACTORS

No information provided

None

Small break LOCA

Feedwater transients

COMPONENT DESCRIPTION

No information provided 
PLANT:

Location:

Vendor:

License Date:

Utility:

Type:

MWt:

MWe:

\# of Bundles:

Group \#:

Code:

Q/A:

Organization:

Admin. Contact:

Technical Contact:

\section{REFERENCES}

Report Number:

Issue Date:

Author:

Title:

Report Number:

Issue Date:

Author:

Title:

Steady States:

Assessments:

Applications:

COMPONENT DESCRIPTION

Core:

Vessel:

Containment:

Balance of Plant:

Primary or Recirc:

Main Steam:

Control Systems:

Auxilliary Systems:

Fuel Rod Behavior:

Kinetics:

\section{Garona}

Spain

$\mathrm{GE}$

$5 / 71$

Centrales Nucleares del Norte, SA

BWR/3 188" ID CONTAINMENT

1380

440

$400 \quad 8 \times 8$

G3

TRAC-EF1/MOD

$\mathrm{Y}$

EG\&G Idaho, Inc.

$\begin{array}{lc}\text { G W Johnsen } & 208-526-9854 \\ \text { R R Schultz } & 208-526-9508\end{array}$

ICAP-00188

2/91

J.V. Lopez, et al

Recirculation Suction Large Break LOCA

Analysis of Santa Maria de Garona

Nuclear Power Plant Using TRAC-BF1(G1J1)

ICAP-00204

$6 / 91$

J.L. Crespo, et al

Assessment of MSIV Full Closure for

Santa Maria de Garona NPP Using TRAC BF1

(G1J1)

$104 \%$ thermal core power

Loss of Load

Large Break LOCA

2 axial levels, 3 radial rings

8 axial levels, 4 radial rings, control

rod guide tube flow paths, core bypass

Pressure boundary

Simulated feed and steam lines

One broken loop, one intact loop

simulated steam line

Recirc pump speed

Core spray, HPCI

None

Point kinetics 
PLANT:

Location:

Vendor:

License Date:

Utility:

Type:

MWt:

MWe:

\# of Bundles:

Group \#:

Code:

Q/A:

Organization:

Admin. Contact:

Technical Contact:

\section{REFERENCES}

Steady States:

Assessments:

Applications:

\section{COMPONENT DESCRIPTION}

Core:

Vessel:

Containment:

Balance of Plant:

Primary or Recirc:

Main Steam:

Control Systems:

Auxiliary Systems:

Fuel Rod Behavior:

Kinetics:
Grand Gulf

Port Gibson, Miss.

GE

$7 / 85$

System Energy Resources, Inc.

BWR/6 238" ID CONTAINMENT 3

3579

1142

$7328 \times 8$

G12

TRAC-BD1/MOD

$\mathrm{N}$

EG\&G Idaho, Inc.

$\begin{array}{ll}\text { G W Johnsen } & 208-526-9854 \\ \text { P D Wheatley } & 208-526-9348\end{array}$

None

Steady state control system used

None

None
8 hydraulic levels

9 axial segments, 4 radial segments, 1 azimuthal segment

Pressure boundary

Feedwater is modeled

Both recirculation loops modeled with 6 nodes per loop. Jet pumps modeled 5 node steam lines, MSIV and SRVs are modeled

turbine flow, turbine outlet flow area, steam extraction

None

None

Power vs. time 
PLANT:

Location:

Vendor:

License Date:

Utility:

Type:

MWt:

MWe:

\# of Bundles:

Group \#:

Code:

Q/A:

Organization:

Admin. Contact:

Technical Contact:

\section{REFERENCES}

Report Number:

Issue Date:

Author:

Title:

Steady States:

Assessments:

Applications:

COMPONENT DESCRIPTION

Core:

Vessel:

Containment:

Balance of Plant:

Primary or Recirc:

Main Steam:

Control Systems:

Auxiliary Systems:

Fuel Rod Behavior:

Kinetics:
Kori 1

Korea

W

1978

Korea Electric Power Corporation

2-Loop

1723

587

$121 \quad 14 \times 14$

W9

RELAP5/MOD2

$\mathrm{Y}$

EG\&G Idaho, Inc.
R R Schultz
208-526-9508
R R Schultz 208-526-9508

NUREG/IA-0030

4/90

Bud-Dong Chong, et al

ASSESSMENT OF RELAP5/MOD2 CODE USING

LOSS OF OFFSITE POWER TRANSIENT DATA OF

KNU \#1 PLANT

77.5\% full power

Plant Blackout

None reported
Single channel, 3 node core

Guide tube modeled, no leak paths

Pressure boundary

No feedtrain

Both loops modeled explicitly

Detailed steam lines

Steam dump, turbine trip

Auxiliary feedwater

None

Point kinetics 
PLANT:

Location:

Vendor:

License Date:

Utility:

Type:

MWt:

MWe:

\# of Bundles:

Group \#:

Code:

Q/A:

Orgxization:

Adisun. Contact:

Technical Contact:

\section{REFERENCES}

Report Number:

Issue Date:

Author:

Title:

Steady States:

Assessments:

Applications:

\section{COMPONENT DESCRIPTION}

Core:

Vessel:

Containment:

Balance of Plant:

Primary or Recirc:

Main Steam:

Control Systems:

Auxiliary Systems:

Fuel Rod Behavior:

Kinetics:
Kori 3

Korea

W

$1 / 85$

Korea Electric Power Corporation

3-Loop

2775

800

$157 \quad 17 \times 17$

W6

RELAP5/MOD3

$\mathrm{Y}$

EG\&G Idaho, Inc.
R R Schultz
208-526-9508
R R Schultz
208-526-9508

ICAP-00213

$\cdots$

Kyun-Tae Kim, et al

ASSESSMENT OF RELAP5/MOD3 VERSION 5M5

USING INADVERTENT SAFETY INJECTION DATA

OF KORI UNIT 3 PLANT

83\% full power

Feedwater Transients

None reported

12 node single channel core

3 channel split downcomer

Guide tube and leakage flows modeled

Pressure boundary

Detailed SGs, no feedtrain

All 3 loops modeled

Steam lines modeled in detail

Feedwater controller modeled

Safety injection system

None

Point kinetics 
PLANT:

Location:

Vendor:

License Date:

Utility:

Type:

MWt:

MWe:

\# of Bundles:

Group \#:

Code:

Q/A:

Organization:

Admin. Contact:

Technical Contact:

\section{REFERENCES}

\section{None}

Steady States:

Assessments:

Applications:

COMPONENT DESCRIPTION

GE

$1 / 84$

3293

1036

G9.

$\mathrm{N}$

None
LaSalle County 1

Seneca, Ill.

Commonwealth Edison Co.

BWR/5 251" ID CONTAINMENT 2c

$7648 \times 8$

TRAC-BD1/MOD

EG\&G Idaho, Inc.

G W Johnsen 208-526-9854

J R Larson 208-526-9609

$85 \%$ full power

Stability Analysis

No information 
PLANT:

Location:

Vendor:

License Date:

Utility:

Type:

MWt:

MVie:

\# of Bundles:

Group \#:

Code:

Q/A:

Organization:

Admin. Contact:

Technical Contact:

\section{REFERENCES}

Report Number:

Issue Date:

Author:

Title:

Steady States:

Assessments:

Applications:
North Anna 2

Mineral, Va.

W

$12 / 80$

Virginia Power Co.

3-Loop

2652

915

$157 \quad 17 \times 17$

W6

RELAP5/MOD2

Y

EG\&G Idaho, Inc.

G W Johnsen 208-526-9854

J D Burtt 208-526-9089

EGG-NTAP-6082

$11 / 82$

J. D. Burtt

AUDIT CALCULATIONS FOR A MAIN STEAM LINE

BREAK IN NORTH ANNA, UNIT 2 USING THE

RELAPS COMPUTER CODE

None

None

Steam Line Breaks

Plant Blackout

Return to Power Transients

COMPONENT DESCRIPTION

Core:

Vessel:

Containment:

Balance of Plant:

Primary or Recirc:

Main Steam:

Control Systems:

Auxiliary Systems:

Fuel Rod Behavior:

Kinetics:

Split core model for return to power calculations. 12 core nodes

4 node split lower plenum, 18 node split downcomer, split upper plenum region

Pressure boundary

Feedwater system modeled

19 nodes per loop.

3 nodes, MSIV modeled. Steam generators:

8 node primary, 8 node secondary

Pressurizer heaters, spray line flow,

RCP speed, feedwater, steam flow.

Accumulator

None

Point kinetics feedback as a function of

five plant parameters. 
PLANT:

Location:

Vendor:

License Date:

Utility:

Type:

MWt:

MWe:

\# of Bundles:

Group \#:

Code:

Q/A:

Organization:

Admin. Contact:

Technical Contact:

\section{REFERENCES}

Report Number:

Issue Date:

Author:

Title:

Report Number:

Issue Date:

Author:

Title:

Report Number:

Issue Date:

Author:

Title:

Steady States:

Assessments:

Applications:
Nuclear One 2

Russellville, Ark.

C-E

3/80

Arkansas Power \& Light Co.

2X4 Loop

2452

858

$177 \quad 16 \times 16$

C7

RELAP5/MOD1.5

$\mathrm{N}$

EG\&G Idaho, Inc.

P D Bayless 208-526-9027

P D Bayless 208-526-9027

EGG-NTAP-6226

4/83

P.D. Bayless

ANALYSIS OF STEAM GENERATOR TUBE

RUPTURES IN ARKANSAS NUCLEAR ONE UNIT 2

EGG-NTAP-6309

9/83

P.D. Bayless

ANALYSIS OF THE JUNE 24, 1980 LOSS OF

OFF-SITE POWER TRANSIENT AT ARKANSAS

NUCLEAR ONE UNIT 2

EGG-SAAM-6415

9/83

P.D. Bayless

ANALYSIS OF THE JANUARY 29, 1980 TURBINE TRIP AT ARKANSAS NUCLEAR ONE UNIT 2

$100 \%$ Power / 100\% Flow

Plant blackout

Turbine trip

Steam Generator Tube Ruptures 
Core:

Vessel:

Containment:

Balance of Plant:

Primary or Recirc:

Main Steam:

Control Systems:

Auxiliary Systems:

Fuel Rod Behavior:

Kinetics:
6 node core, 6 node bypass

3 node lower plenum, 10 node downcomer, 5 node upper plenum/upper head region.

Pressure boundary

Main feedwater is modeled

Both loops modeled in detail

Both steam lines modeled in detail

Emergency feedwater, HPCI, charging and let down systems are included.

None

Power vs. time tables 
PLANT:

Location:

Vendor:

License Date:

Utility:

Type:

MWt:

MWe:

\# of Bundles:

Group \#:

Code:

Q/A:

Organization:

Admin. Contact:

Technical Contact:

\section{REFERENCES}

Report Number:

Issue Date:

Author:

Title:

Report Number:

Issue Date:

Author:

Title:

Report Number:

Issue Date:

Author:

Title:

Steady States:

Assessments:

Applications:
Oconee 1

Seneca, S.C.

B\&W

7/73

Duke Power Co.

2-Loop Low

2452

846

$177 \quad 15 \times 15$

B3

RELAP5/MOD2

$\mathrm{Y}$

EG\&G Idaho, Inc.

G W Johnsen 208-526-9854

C D Fletcher 208-526-9859

EGG-NTAP-6190

$3 / 83$

M. A. Bolander, et. al.

RELAP5 ANALYSIS OF OCONEE-1 PRESSURIZED

WATER REACTOR TRANSIENTS FOR THE

PRESSURIZED THERMAL SHOCK INTEGRATION

STUDY

NUREG/CR-3761

6/84

C. D. Fletcher, et. al.

RELAP5 THERMAL-HYDRAULIC ANALYSES OF

PRESSURIZED THERMAL SHOCK SEQUENCES FOR

THE OCONEE-1 PRESSURIZED WATER REACTOR

EGG-RTH-7066

10/85

M. A. Bolander

RELAPS THERMAL-HYDRAULIC ANALYSES FOR

TWO PRESSURIZED THERMAL SHOCK SEQUENCES

FOR THE OCONEE-1 PWR

$100 \%$ Power / $100 \%$ Flow

None

Steam Line Breaks

Feedwater Transients

Small Break LOCA

Steam Generator Tube Ruptures 
Core:

Vessel:

Containment:

Balance of Plant:

Primary or Recirc:

Main Steam:

Control Systems:

Auxiliary Systems:

Fuel Rod Behavior:

Kinetics:
6 Node core, 6 Node bypass

2 node lower plenum, 9 node downcomer, 10 node upper plenum/ upper head region Pressure boundary

Feedwater and turbine valves

Both loops modelled. 27 Nodes per loop.

Pressurizer spray line is included

Steam lines, MSIV, turbine stop valves, turbine bypass valves are modeled $B \& W$ integrated control system (ICS) is modeled.

HPI, LPI, Accumulators, emergency feedwater

None

Input power table only 
PLANT:

Location:

Vendor:

License Date:

Utility:

Type:

MWt:

MWe:

\# of Bundles:

Group \#:

Code:

Q/A:

Organization:

Admin. Contact:

Technical Contact:

\section{REFERENCES}

Report Number:

Issue Date:

Author:

Title:

Steady States:

Assessments:

Applications:
Oconee 1

Seneca, S.C.

B\&W

7/73

Duke Power Co.

2-Loop Low

2452

846

$177 \quad 15 \times 15$

B3

RELAP5/SCDAP/MOD3

Y

EG\&G Idaho, Inc.

G W Johnsen 208-526-9854

J C Determan 208-526-2888
EGG-EAST-9793

$7 / 91$

J.C. Determan, et al

DEVELOPEMENT OF A SCDAP/RELAP5/MOD3

MODEL OF OCONEE 1 FOR USE WITH THE

NUCLEAR PLANT ANALYZER

$100 \%$ Power / 100\% Flow

None

Large Break LOCA

Small Break LOCA

Single channel, six-node core

2 node lower plenum,

10 node upper plenum/upper head region

Pressure boundary

Feedwater and turbine valves

Both loops modelled.

Pressurizer spray line is included

Steam lines, MSIV, turbine stop valves, turbine bypass valves are modeled

Control Systems:

Auxiliary Systems:

Fuel Rod Behavior:

Kinetics:
$B \& W$ integrated control system (ICS) is modeled.

HPI, LPI, Accumulators, emergency feedwater

SCDAP input for single channel core

Point Kinetics 
PLANT:

Location:

Vendor:

License Date:

Utility:

Type:

MWt:

MWe:

\# of Bundles:

Group \#:

Code:

Q/A:

Organization:

Admin. Contact:

Technical Contact:

\section{REFERENCES}

Report Number:

Issue Date:

Author:

Title:

Report Number:

Issue Date:

Author:

Title:

Report Number: Issue Date:

Author:

Title:

Report Number:

Issue Date:

Author:

Title:
Oconee 1

Seneca, S.C.

B\&W

7/73

Duke Power Co.

2-Loop Low

2452

846

$17715 \times 15$

B3

TRAC-PF1/MOD1

Y

Los Alamos National Laboratory

M W Cappiello 505-667-4097

M W Cappiello 505-667-4097

NUREG/CR-3706

$5 / 85$

B. Bassett, et. al.

TRAC ANALYSES OF SEVERE OVERCOOLING

TRANSIENTS FOR THE OCONEE-1 PWR

NUREG/CR-4140

$4 / 85$

DOMINANT ACCIDENT SEQUENCES IN OCONEE-1 PRESSURIZED WATER REACTOR

LA-UR-86-1310

$4 / 86$

Henry J. Stumpf

USE OF TURBINE BYPASS VALVES TO CODE AND

DEPRESSURIZE OCONEE-1 FOLLOWING A

SMALL-BREAK LOSS-OF-COOLANT ACCIDENT

WITH FAILURE OF THE HIGH PRESSURE

INJECTION SYSTEM

LA-UR-84-3284

1984

B. Nassersharif

INTERFACING SYSTEMS LOSS-OF-COOLANT ACCIDENT IN OCONEE-1 PRESSURIZED WATER REACTORS 
Report Number:

Issue Date:

Author:

Title:

Report Nurnber: Issue Date:

Author:

Title:

Report Number: Issue Date:

Author:

Title:

Report Number: Issue Date:

Author:

Title:

Report Number: Issue Date:

Author:

Title:

Report Number: Issue Date:

Author:

Title:

Report Number: Issue Date:

Author:

Title:

Report Number: Issue Date:

Author:
LA-UR-84-2721

1984

J. Dearing

ANALYSIS OF SMALL-SMALL BREAK LOCA SEQUENCES FOR THE OCONEE-1 PWR

LA-UR-84-2640

1984

B. Nassersharif

INTERFACING SYSTEMS LOSS-OF-COOLANT

ACCIDENT IN OCONEE-1 PRESSURIZED WATER

REACTOR

LA-UR-84-2309

1984

B. Boyack

AN ASSESSMENT OF THE UTILITY OF FEED-AND-BLEED OPERATING MAPS (OCONEE-1)

LA-UR-84-2078

1984

R. Henninger, et al "LOFW-INITIATED ATWS FOR OCONEE-1 USING TRAC-PF1/MOD1

LA-UR-84-2077

1984

B. Nassersharif

INTERFACING SYSTEMS LOSS-OF-COOLANT ACCIDENT IN OCONEE-1 PRESSURIZED WATER REACTOR

LA-UR-83-3182

1983

J. Ireland

TRAC ANALYSES OF SEVERE OVERCOOLING TRANSIENTS FOR THE OCONEE-1 PWR

LA-UR-83-3011

$10 / 83$

J. Ireland

TRAC ANALYSIS AND SUPPORT OF OCONEE-1 PTS STUDIES

LA-UR-83-2894

$10 / 83$

R. Henninger 
Title:

Report Number: Issue Date:

Author:

Title:

Report Number: Issue Date:

Author:

Title:

Report Number: Issue Date: Author:

Title:

Report Number: Issue Date:

Author:

Title:

Report Number: Issue Date:

Author:

Title:

Report Number: Issue Date:

Author:

Title:

Report Number: Issue Date: Author:
LOSS-OF-FEEDWATER TRANSIENTS FOR OCONEE-1

LA-UR-83-2888

1983

J. Dearing

ANALYSIS OF SMALL-SMALL BREAK LOCA SEQUENCES IN THE OCONEE-1 PLANT

LA-UR-83-2883

1983

R. Henninger COMBINED SECONDARY-SIDE BREAK/LOSS-OF-FEEDWATER TRANSIENTS FOR OCONEE-1

LA-UR-83-2816

$9 / 83$

M. Burkett, et al TRAC-PF1- PRESSURIZED THERMAL SHOCK

CALCULATIONS FOR SEVERAL SMALL-BREAK-LOSS-OF-COOLANT ACCIDENT TRANSIENTS IN THE OCONEE-1 NUCLEAR POWER PLANT

LA-UR-83-2346

$8 / 83$

J. Ireland

TRAC ANALYSIS AND SUPPORT OF OCONEE-1 PTS STUDIES

LA-UR-83-2288

1983

J. Dearing

ANALYSIS OF SMALL-SMALL-BREAK LOCA SEQUENCES IN THE OCONEE-1 POWER PLANT

LA-UR-83-0960

$3 / 83$

B. Bassett

TRAC THERMAL-HYDRAULIC ANALYSIS OF THE OCONEE-1 PWR FOR THE PRESSURIZED THERMAL SHOCK PROJECTS

LA-TCN-TN-83-1

1983

J. Ireland 
Title:

Report Number:

Issue Date:

Author:

Title:

Steady States:

Assessments:

Applications:
TRAC ANALYSIS OF SEVERE OVERCOOLING TRANSIENTS FOR THE OCONEE-1 PWR

LA-UR-82-3720

$12 / 82$

M. W. Burkett

TRAC-PF1 PRESSURIZED THERMAL SHOCK

CALCULATION FOR SEVERAL SMALL BREAK TRANSIENTS IN THE OCONEE-1 NUCLEAR POWER PLANT

100\% Power/ $100 \%$ Flow

Turbine Trip

Large Break LOCA

Feed and Bleed Recovery

COMPONENT DESCRIPTION

Core:

Vessel:

Containment:

Balance of Plant:

Primary or Recirc:

Main Steam:

Control Systems:

Auxiliary Systems:

Fuel Rod Behavior:

Kinetics:
6 axial levels, 6 theta segments, 1 radial ring

14 axial levels, 2 radial rings,

6 azimuthal segments

Pressure boundary

Main feedwater is modeled

Both loops modeled in detail

Pressure boundary

Basic plant protection

HPI, LPI, accumulators

Detailed reflood mode

Input power table only 
PLANT:

Location:

Vendor:

License Date:

Utility:

Type:

MWt:

MWe:

\# of Bundles:

Group \#:

Code:

Q/A:

Organization:

Admin. Contact:

Technical Contact:

\section{REFERENCES}

Report Number:

Issue Date:

Author:

Title:

Steady States:

Assessments:

Applications:

COMPONENT DESCRIPTION

Core:

Vessel:

Containment:

Balance of Plant:

Primary or Recirc:

Main Steam:

Control Systems:

Auxilliary Systems:

Fuel Rod Behavior:

Kinetics:
Phillipsburg 2

Germany

KWU

$\div / 85$

Keinkraftwerk Phillipsburg

4-Loop

3765

1300

$19316 \times 16$

W2

\section{RELAP5/MOD2}

$Y$

EG\&G Idaho, Inc.

G W Johnsen 208-526-9854

R R Schultz 208-526-9508

NUREG/IA-0057

4/92

G. Gerth

Assessment Study of RELAP5/MOD2 Cycle 36.04 Based on the Commissioning Test

Reactor Trip at Full Load at the

Phillipsburg 2 Nuc!ear Power Plant

$100 \%$ power

Plant Trip

None reported
Single channel, 8 level core

Core bypass and upper head leakage paths

Pressure boundary

Detailed SG, no feedtrain

One triple loop, one single loop, simple pressurizer

Detailed steamline

No information

No information

None

Point kinetics 
PLANT:

Location:

Vendor:

License Date:

Utility:

Type:

MWt:

MWe:

\# of Bundles:

Group \#:

Code:

Q/A:

Organization:

Admin. Contact:

Technical Contact:

\section{REFERENCES}

Report Number:

Issue Date:

Author:

Title:

Report Number:

Issue Date:

Author:

Title:

Report Number:

Issue Date:

Author:

Title:

Report Number:

Issue Date:

Author:

Title:

Steady States:

Assessments:
R. E. Ginna

Ontario, N.Y.

W

7/70

Rochester Gas \& Electric Corp.

2-Loop

1300

470

$121 \quad 14 \times 14$

W9

TRAC-PF1/MOD1

Y

Los Alamos National Laboratory

J F Lime 505-667-4097

J F Lime $\quad 505-667-4097$

LA-UR-86-2818

$8 / 86$

J. F. Lime, et al

THE GINNA TUBE-RUPTURE EVENT : A

PLANT-TRANSIENT BENCHMARK FOR TRAC

LA-UR-86-0205

$1 / 86$

J. F. Lime, et al

RAPID-RESPONSE ANALYSIS OF THE

DAVIS-BESSE LOSS-OF-FEEDWATER EVENT OF

JUNE 9, 1985

LA-UR-85-3117

$8 / 85$

R. P. Jenks, et al

PRELIMINARY TRAC CALCULATION OF R. E.

GINNA STEAM GENERATOR TUBE-RUPTURE EVENT

ON JANUARY 25, 1982

NUREG/CR-4988

9/87

J. F. Lime, et al

A TRAC-PF1/MOD1 ANALYSIS OF THE GINNA TUBE-RUPTURE EVENT ON JANUARY 25, 1982

$100 \%$ core power

Steam Generator Tube Ruptures 
Applications:

Steam Generator Tube Ruptures

\section{COMPONENT DESCRIPTION}

Core:

Vessel:

1 radial ring, 4 azimuthal sectors

2 radial rings, 4 azimuthal sectors,

12 axial levels

Containment:

Balance of Plant:

Primary or Recirc:

Main Steam:

Control Systems:

Auxiliary Systems:

Fuel Rod Behavior:

Kinetics:

Pressure boundary

both loops modeled individually turbine stop valve, steam dump SG level, PORV control, turbine trips, main feedwater trips,

Auxiliary feedwater

8 radial nodes, conduction and reflood

Input power table only 
PLANT:

Location:

Vendor:

License Date:

Utility:

Type:

MWt:

MWe:

\# of Bundles:

Group \#:

Code:

Q/A:

Organization:

Admin. Contact:

Technical Contact:

\section{REFERENCES}

Report Number:

Issue Date:

Author:

Title:

Report Number:

Issue Date:

Author:

Title:

Steady States:

Assessments:

Applications:
RESAR

N/A

$\mathrm{W}$

N/A

N/A

4-Loop

N/A

N/A

$19317 \times 17$

W2

RELAP5/MOD2

$\mathrm{Y}$

EG\&G Idaho, Inc.

$\begin{array}{lc}\text { G W Johnsen } & 208-526-9854 \\ \text { J M Cozzoul } & 208-526-9901\end{array}$

NUREG/CR-4384

$3 / 86$

BREAK SPECTRUM ANALYSIS FOR SMALL BREAK LOSS OF COOLANT ACCIDENTS IN A RESAR-3S PLANT

EGG-NTAP-6032

9/82

\section{BEST ESTIMATE ANALYSIS OF A SMALL BREAK LOCA IN A RESAR-3S PRESSURIZED WATER REACTOR}

$100 \%$ power, $100 \%$ Flow

None

Small Break LOCA

Feed and bleed recovery

\section{COMPONENT DESCRIPTION}

Core:

Vessel:

Containment:

Balance of Plant:

Primary or Recirc:

Main Steam:

Control Systems:

Auxiliary Systems:

Fuel Rod Behavior:

Kinetics:
Six node, single channel core

Guide tube bypass modeled

iressure boundary

Detailed steam generators, no feedtrain

3 lumped loops, 1 explicit loop

Pressure boundary

None

ECCS modeled on lumped loop

None

Input power table only 
PLANT:

Location:

Vendor:

License Date:

Utility:

Type:

MWt:

MWe:

\# of Bundles:

Group \#:

Code:

Q/A:

Organization:

Admin. Contact:

Technical Contact:

\section{REFERENCES}

Report Number:

Issue Date:

Author:

Title:

Report Number:

Issue Date:

Author:

Title:

Report Number:

Issue Date:

Author:

Title:

Report Number:

Issue Date:

Author:

Title:
RESAR

N/A

W

N/A

N/A

4-Loop

N/A

N/A

$19317 \times 17$

W2

TRAC-PF1/MOD2

Y

Los Alamos National Laboratory

J W Spore 505-667-2125

J W Spore 505-667-2125
LA-UR-85-0858

3/85

R. Fujita, et al

TRAC-PF1 ANALYSIS OF A BEST-ESTIMATE

LARGE-BREAK LOCA IN A WESTINGHOUSE PWR

WITH FOUR LOOPS AND 17X17 FUEL

LA-UR-84-2056

1984

J. F. Lime

LOSS-OF-FEEDWATER/FEED AND BLEED IN A WESTINGHOUSE THREE-LOOP, LOW-HEAD SAFETY INJECTION PWR

LA-UR-84-0109

$1 / 84$

E. Barts, et al

TRAC-PF1 STUDY OF LOSS OF PUMIP SEALS IN

A WESTINGHOUSE PWR

LA-UR-85-3117

$8 / 85$

R. P. Jenks, et al PRELIMINARY TRAC CALCULATION OF R. E. GINNA STEAM GENERATOR TUBE-RUPTURE EVENT ON JANUARY 25, 1982 
Report Number:

Issue Date:

Author:

Title:

Report Number:

Issue Date:

Author:

Title:

Report Number:

Issuc Date:

Author:

Title:

Assessments:

Applications:

COMPONENT DESCRIPTION

Core:

Vessel:

Containment:

Balance of Plant:

Primary or Recirc:

Main Steam:

Control Systems:

Auxiliary Systems:

Fuel Rod Behavior:

Kinetics:
LA-UR-82-3237

$11 / 82$

J. F. Lime

TRAC-PD2 CALCULATIONS OF A

MAIN-STEAM-LINE BREAK IN A WESTINGHOUSE

PRESSURIZED WATER REACTOR-COOLANT PUMP

TRIP

LA-UR-82-3235

$11 / 82$

J. F. Lime

TRAC-PD2 CALCULATION OF A COLD-LEG SMALL BREAK IN A WESTINGHOUSE FOUR-LOOP

PRESSURIZED WATER REACTOR

NUREG/CR-3312

$5 / 83$

J. E. Elliott

EFFECTS OF THE REACTOR CODLANT PUMPS

FOLLOWING A SMALL BREAK IN A

WESTINGHOUSE PWR

None

Large Break LOCA
17 axial levels, 4 radial rings,

8 theta segments

Pressure boundary

Feedwater modeled

All four loops modeled

Steam line modeled

Basic plant protection for LBLOCA

HPI, LPI, accumulator

None

Point Kinetics 
PLANT:

Location:

Vendor:

License Date:

Utility:

Type:

MWt:

MWe:

\# of Bundles:

Group \#:

Code:

Q/A:

Organization:

Admin. Contact:

Technical Contact:

\section{REFERENCES}

Report Number:

Issue Date:

Author:

Title:

Steady States:

Assessments:

Applications:

COMPONENT DESCRIPTION

Core:

Vessel:

Containment:

Balance of Plant:

Primary or Recirc:

Main Steam:

Control Systems:

Auxilliary Systems:

Fuel Rod Behavior:

Kinetics:
Ringhals 2

Sweden

W

$5 / 75$

Statens Vattenfallsverk

3-Loop

2440

800

$157 \quad 15 \times 15$

W7

TRAC-PF1/MOD1

$\mathrm{Y}$

EG\&G Idaho, Inc.

G W Johnsen 208-526-9854

R R Schultz 208-526-9508

ICAP-00145

2/88

F. Pelayo, et al

Assessment of TRAC-PF1/MOD1 Against an Inadvertent Steam Line Isolation Valve

Closure in the Ringhals 2 Power Plant

$80 \%$ core power

Loss of Load

None reported
5 axial leveis, average core and hot rod

1-D vessel, core bypass, upper head leakage

Pressure boundary

Detailed SG, no feedtrain

2 double loop one single loop

detailed pressurizer

Detaled steamlines

Pressurizer pressure and level, steam

line break protection

Detailed accumulator, HPSI, LPSI, CVCS

None

Point kinetics 
PLANT:

Location:

Vendor:

License Date:

Utility:

Type:

MWt:

MWe:

\# of Bundles:

Group \#:

Code:

Q/A:

Organization:

Admin. Contact:

Technical Contact:

\section{REFERENCES}

Report Number: Issue Date:

Author:

Title:

Report Number:

Issue Date:

Author:

Title:

Steady States:

Assessments:

Applications:

\section{COMPONENT DESCRIPTION}

Core:

Vessel:

Containment:

Balance of Plant:

Primary or Recirc:

Main Steam:

Control Systems:

Auxilliary Systems:

Fuel Rod Behavior:

Kinetics:

\section{Ringhals 4}

Sweden

W

$11 / 83$

Statens Vattenfallsverk

3-Loop

2775

915

$157 \quad 17 \times 17$

W6

TRAC-PF1/MOD1

Y

EG\&G Idaho, Inc.
G W Johnsen
208-526-9854
R R Schultz 208-526-9508

NUREG/IA-0038

$3 / 92$

A. Sjoberg

Assessment of TRAC-PF1/MOD1 Against an Inadvertent Feedwater Line Isolation

Transient in the Ringhals 4 Power Plant

ICAP-00053

$3 / 87$

A. Sjoberg

Assessment of TRAC-PF1/MOD1 Against a

Loss of Grid Transient in Ringhals 4

Power Plant

$100 \%$ core power

Plant Blackout

Feedwater Transients

None reported

1-D, seven levels

1-D, core bypass, basic nodalization

Pressure boundary

Detailed SG, no feedtrain

All 3 loops lumped into one, detailed pressurizer detailed steamline

Pressurizer pressure and level, scram turbine trip and SG aux feed on SG level

None

None

Point Kinetics 
PLANT:

Location:

Vendor:

License Date:

Utility:

Type:

MWt:

MWe:

\# of Bundles:

Group \#:

Code:

Q/A:

Organization:

Admin. Contact:

Technical Contact:

\section{REFERENCES}

Report Number:

Issue Date:

Author:

Title:

Report Number:

Issue Date:

Author:

Title:

Steady States:

Assessments:

Applications:
Robinson 2

Hartsville, S.C.

W

$3 / 71$

Carolina Power \& Light Co.

3-Loop

2094

665

$15715 \times 15$

W7

RELAP5/MOD2

Y

EG\&G Idaho, Inc.
G W Johnsen 208-526-9854
C D Fletcher 208-526-9859

NUREG/CR-3977

4/85

C. D. Fletcher, et. al.

RELAP5 THERMAL-HYDRAULIC ANALYSES OF PRESSURIZED THERMAL SHOCK SEQUENCES FOR THE H. B. ROBINSON UNIT 2 PRESSURIZED WATER REACTOR

NUREG/CR-3935

$5 / 85$

C. D. Fletcher, et. al.

THERMAL-HYDRAULIC ANALYSES OF OVERCOOLING SEQUENCES FOR THE H. B. ROBINSON UNIT 2 PRESSURIZED THERMAL SHOCK STUDY

$2300 \mathrm{MWt}$ (rated), $2200 \mathrm{MWt}$, Hot Standby

Plant Trip

Small Break LOCA

Steam Line Breaks

Steam Generator Tube Ruptures

Stuck Open PORV

Feedwater Transients

Feed and Bleed Recovery 
Core:

Vessel:

Containment:

Balance of Plant:

Primary or Recirc:

Main Steam:

Control Systems:

Auxiliary Systems:

Fuel Rod Behavior:

Kinetics:
Six node average core and six node bypass

3 Node Lower Plenum, 12 Node downcomer Seven Node Upper Plenum/ Upper head

Pressure boundary

Feedwater and Turbines modelled

All three loops modelled explicitly, 130 total volumes

Steam lines are modelled to the condenser

Steam dump, SG level, pressurizer level and pressure

HPI, LPI, Makeup, Accumulator, Auxiliary Feedwater

None

Input power table only 


\section{PLANT:}

Location:

Vendor:

License Date:

Utility:

Type:

MWt:

MWe:

\# of Bundles:

Group \#:

Code:

Q/A:

Organization:

Admin. Contact:

Technical Contact:

\section{REFERENCES}

Steady States:

Assessments:

Applications:

\section{COMPONENT DESCRIPTION}

Core:

Vessel:

Containment:

Balance of Plant:

Primary or Recirc:

Main Steam:

Control Systems:

Auxiliary Systems:

Fuel Rod Behavior:

Kinetics:
Robinson 2

Hartsville, S.C.

W

$3 / 71$

Carolina Power \& Light Co.

3-Loop

2094

665

$157 \quad 15 \times 15$

W7

RELAP5/MOD3

$\mathrm{N}$

EG\&G Idaho, Inc.

G W Johnsen 208-526-9854

P A Roth 208-526-9188

None

$2300 \mathrm{MWt}$ (rated)

None

None
Six node average core and six node bypass

3 Node Lower Plenum, 12 Node downcomer

Seven Node Upper Plenum/ Upper head

Pressure boundary

Feedwater and Turbines modelled

All three loops modelled explicitly,

130 total volumes

Steam lines are modelled to the

condenser

Steam dump, SG level, pressurizer level

and pressure

HPI, LPI, Makeup, Accumulator,

Auxiliary Feedwater

None

Input power table only 
PLANT:

Location:

Vendor:

License Date:

Utility:

Type:

MWt:

MWe:

\# of Bundles:

Group \#:

Code:

Q/A:

Organization:

Admin. Contact:

Technical Contact:

\section{REFERENCES}

Report Number:

Issue Date:

Author:

Title:

Steady States:

Assessments:

Applications:
Robinson 2

Hartsville, S.C.

W

$3 / 71$

Carolina Power \& Light Co.

3-Loop

2094

665

$157 \quad 15 \times 15$

W7

TRAC-PF1/MOD1

Y

Los Alamos National Laboratory

J F Lime 505-667-4097

J F Lime 505-667-4097
LA-UR-84-2101

$6 / 84$

J. F. Lime

A PRIMARY-SYSTEM FEED-AND-BLEED

TRANSIENT IN A THREE-LOOP PRESSURIZED

WATER REACTOR FOLLOWING A COMPLETE LOSS

OF FEEDWATER WITH DELAYED REACTOR TRIP

$100 \%$ core power

None

Small Break LOCA

Steam Generator Tube Ruptures

Feed and Bleed Recovery

\section{COMPONENT DESCRIPTION}

Core:

1 radial ring, 6 azimuthal sectors,

4 axial levels

Vessel:

2 radial rings, 6 azimuthal sectors,

12 axial levels

Pressure boundary

Steam generators modeled in detail

All loops modeled individually

Turbine stop valves and steam dump

valves modeled

Control Systems:

Auxiliary Systems:

Fuel Rod Behavior:

Kinetics:
SG level, PORV, main feedwater, aux. feedwater, pressurizer pressure pressurizer heater control

8 radial nodes in fuel, gap, and

cladding

Input power table only 
PLANT:

Location:

Vendor:

License Date:

Utility:

Type:

MWt:

MWe:

\# of Bundles:

Group \#:

Code:

Q/A:

Organization:

Admin. Contact:

Technical Contact:

\section{REFERENCES}

Report Number:

Issue Date:

Author:

Title:

Steady States:

Assessments:

Applications:

\section{COMPONENT DESCRIPTION}

Core:

Vessel:

Containment:

Balance of Plant:

Primary or Recirc:

Main Steam:

Control Systems:

Auxiliary Systems:

Fuel Rod Behavior:

Kinetics:
Seabrook

Seabrook, N.H.

W

190

New Hampshire Yankee

4-Loop

3425

1150

$\begin{array}{ll}193 & 17 \times 17\end{array}$

W2

RELAP5/MOD2

$\mathrm{Y}$

EG\&G Idaho, Inc.
P D Bayless 208-526-9027
P D Bayless 208-526-9027

\section{EGG-NTP-6700}

9/84

P. D. Bayless, et. al.

ANALYSIS OF A STA'TION BLACKOUT TRANSIENT

AT THE SEABROOK NUCLEAR POWER PLANT

$100 \%$ Power/ $100 \%$ Flow

None

Plant Blackout

Severe Accident Scenarios

2 core models: 1) single channel 6 nodes

2) three channel with crossflow

3 node lower plenum, 11 node downcomer,

9 node upper plenum/ upper head

Pressure boundary

Feedwater modeled

Three loops lumped into one, plus one

single loop.

MSIV and SRV modeled

None

None

None

Power vs. time table 
PLANT:

Location:

Vendor:

License Date:

Utility:

Type:

MWt:

MWe:

\# of Bundles:

Group \#:

Code:

Q/A:

Organization:

Admin. Contact:

Technical Contact:

\section{REFERENCES}

Report Number:

Issue Date:

Author:

Title:

Steady States:

Assessments:

Applications:

\section{COMPONENT DESCRIPTION}

Core:

Vessel:

Containment:

Balarye of Plant:

Primary or Recirc:

Main Steam:

Control Systems:

Auxiliary Systems:

Fuel Rod Behavior:

Kinetics:
Seabrook

Seabrook, N.H.

W

190

New Hampshire Yankee

4-Loop

3425

1150

$193 \quad 17 \times 17$

W2

RELAP5/SCDAP/MOD3

$\mathrm{Y}$

EG\&G Idaho, Inc.
$\mathrm{K} R$ Jones
208-526-0809
$\mathrm{K} R$ Jones
208-526-0809

NUREG/CR-5787

3/92

K. R. Jones, et al

TIMING ANALYSIS OF PWR FUEL PIN FAILURE

$100 \%$ Power/ $100 \%$ Flow

None

Severe Accident Scenarios
3 channel, 9 node core

2 channel split downcomer

Pressure boundary

F eedwater modeled

Three loops lumped into one, plus one single loop.

MSIV and SRV modeled

None

None

SCDAP fuel model used

Point kinetics 
PLANT:

Location:

Vendor:

License Date:

Utility:

Type:

MWt:

MWe:

\# of Bundles:

Group \#:

Code:

Q/A:

Organization:

Admin. Contact:

Technical Contact:

\section{REFERENCES}

Report Number:

Issue Date:

Author:

Title:

Steady States:

Assessinents:

Applications:
SNUPPS

N/A

W

N/A

N/A

4-Loop

N/A

N/A

$19317 \times 17$

W2

RELAP5/MOD2

$\mathrm{N}$

EG\&G Idaho, Inc.

G W Johnsen 208-526-9854

C M Kullberg 208-526-9565

NUREG/CR-5557

$4 / 90$

C.M. Kullberg

RELAP5 Thermal-Hydraulic Analysis of the SNUPPS Pressurized Water Reactor

$100 \%$ power, $100 \%$ flow

None

Plant Blackout

Small Break LOCA

Steam Line Breaks

Steam Generator Tube Ruptures

Feedwater Transients

\section{COMPONENT DESCRIPTION}

Core:

Vessel:

Containment:

Balance of Plant:

Primary or Recirc:

Main Steam:

Control Systems:

Auxiliary Systems:

Fuel Rod Behavior:

Kinetics:
Single channel, 3 node core Guide tube flow and leakages modeled Pressure boundary Detailed feedtrain and SGs

All four loops modeled Detailed steam lines modeled None Accumulator, HPI, LPI modeled None Input power table only 
PLANT:

Location:

Vendor:

License Date:

Utility:

Type:

MWt:

MWe:

\# of Bundles:

Group \#:

Code:

Q/A:

Organization:

Admin. Contact:

Technical Contact:

\section{REFERENCES}

Report Number:

Issue Date:

Author:

Title:

Steady States:

Assessments:

Applications:
Surry 1

Gravel Neck, Va.

W

$12 / 72$

Virginia Power Co.

3-Loop

2441

781

$157 \quad 15 \times 15$

W7

RELAP5/SCDAP/MOD2

$\mathrm{Y}$

EG\&G Idaho, Inc.
P D Bayless
208-526-9027
P D Bayless 208-526-9027

NUREG/CR-5214

$10 / 88$

P. D. Bayless

ANALYSES OF NATURAL CIRCULATION DURING A SURRY STATION BLACKOUT USING SCDAP/

RELAP5

$12 \%$ core power

None

Severe Accident Scenarios

Plant Blackout

\section{COMPONENT DESCRIPTION}

Core:

2 core models: 1) Single channel, 10

node core 2) 3 channel, 10 node core

Vessel:

2 node lower plenum, 11 node downcomer.

8 node upper plenum/ upper head

Containment:

Pressure boundary

Feedwater modeled

All three loops modeled

One node steam line with MSIV, PORV and

Safety valves

None

Auxiliary feedwater, HPI, LPI,

Accumulator

SCDAP used

Point kinetics. 
PLANT:

Location:

Vendor:

License Date:

Utility:

Type:

MWt:

MWe:

\# of Bundles:

Group \#:

Code:

Q/A:

Organization:

Admin. Contact:

Technical Contact:

\section{REFERENCES}

Report Number:

Issue Date:

Author:

Title:

Report Number:

Issue Date:

Author:

Title:

Report Number:

Issue Date:

Author:

Title:

Repcrt Number: Issue Date:

Author:

Title:

Report Number:

Issue Date:

Author:

Title:
Three Mile Island 1

Londonderry Twp., Pa.

B\&W

9/74

GPU Nuclear Corp.

2-Loop Low

2452

808

$177 \quad 15 \times 15$

B3

TRAC-PF1/MOD1

Y

Los Alamos National Laboratory

B B Boyack 505-667-4097

B B Boyack 505-667-4097

LA-UR-89-2115

6/89

Frank Motley

NATURAL CIRCULATION AND STRUCTURAL HEATUP IN TMI-2 AFTER CORE RECOVERY

LA-UR-88-3348

$10 / 88$

Frank Motley, et al

MODELING OF THE TMI-2 ACCIDENT WITH

MELPROG/TRAC AND CALCULATION RESULTS FOR PHASES 1 AND 2

LA-UR-88-1364

$4 / 88$

Richard Jenks

PRELIMINARY MODELING OF THE TMI-2

ACCIDENT WITH MELPROG/TRAC

LA-UR-85-1404

$8 / 85$

B. Nassersharif

ANALYSIS OF MULTIPLE-TUBE RUPTURE IN

BOTH STEAM GENERATORS FOR THE THREE MILE

ISLAND-1 PRESSURIZED WATER REACTOR

LA-UR-85-0946

1985

B. Nassersharif

ANALYSIS OF MULTIPLE TUBE RUPTURES IN BOTH STEAM GENERATORS FOR THE THREE MILE ISLAND-1 PRESSURIZED WATER REACTOR. (NEWPORT) 
Report Number: Issue Date:

Author:

Title:

Report Number: Issue Date:

Author:

Title:

Report Number: Issue Date:

Author:

Title:

Report Number: Issue Date:

Author:

Title:

Report Number: Issue Date:

Author:

Title:

Report Number: Issue Date:

Author:

Title:

Report Number: Issue Date:

Author:

Title:
LA-UR-85-0355

$1 / 85$

B. Nassersharif

ALTERNATE STEAM GENERATOR TUBE RUPTURE MITIGATION STRATEGIES FOR THE THREE MILE ISLAND-1 PRESSURIZED WATER REACTOR

DURING A LOSS-OF-OFFSITE POWER

LA-UR-85-0182

1985

B. Nassersharif

ALTERNATE STEAM GENERATOR TUBE RUPTURE MITIGATION STRATEGIES FOR THE THREE MILE ISLAND UNIT 1 DURING A LOSS-OF-OFFSITE POWER

LA-UR-85-3555

1985

B. Nassersharif

ALTERNATE STEAM GENERATOR TUBE RUPTURE MITIGATION STRATEGIES FOR THE THREE MILE ISLAND UNIT 1 DURING A LOSS-OF-OFFSITE POWER (DENVER PAPER)

LA-UR-84-3588

1984

B. Boyack

ALTERNATIVE STEAM GENERATOR TUBE RUPTURE MITIGATION ON STRATEGIES FOR THE THREE MILE ISLAND-1 PRESSURIZED WATER REACTOR DURING A LOSS-OF-OFFSITE POWER

LA-UR-84-2586

1984

B. Nassersharif ANALYSIS OF MULTIPLE TUBE RUPTURES IN BOTH STEAM GENERATORS FOR THE THREE MILE ISLAND-1 PRESSURIZED WATER REACTOR

LA-UR-84-3465

1984

B. Nassersharif

ALTERNATE STEAM GENERATOR TUBE RUPTURE MITIGATION STRATEGIES FOR THE THREE MILE ISLAND-1 PRESSURIZED WATER REACTOR DURING A LOSS-OF-OFFSITE POWER

LA-UR-83-2335

$8 / 83$

E. Schwegler

FUEL MODELS AND RESULTS FROM THE

TRAC-PF1/MIMAS TMI-2 ACCIDENT 


\section{CALCULATION}

Report Number: Issue Date: Author:

Title:

Report Number: Issue Date:

Author:

Title:

Report Number: Issue Date:

Author:

Title:

Report Number: Issue Date: Author:

Title:

Report Number. Issue Date: Author: Ti'le:

Report Number: Issue Date: Author:

Title:

Report Number: Issue Date:

Author:

Title:

Report Number: Issue Date:

Author:

Title:

Report Number: Issue Date:
LA-UR-83-1743

1983

R. Henninger, et al TRAC-PF1 FEED-AND-BLEED CALCULATIONS FOR TMI-1 ANS PAPER

LA-UR-83-0963

3/83

E. C. Schwegler

FUEL MODELS AND RESULTS FROM THE

TRAC-PF1/MIMAS TMI-2 ACCIDENT

CALCULATION

LA-UR-83-0904

1983

P. J. Maudlin

INTEGRATED ANALYSIS OF THE TMI-2

ACCIDENT

LA-UR-83-0737

1983

P. Maudlin, et al

DAMAGE ASSESSMENT OF TMI-2

LA-UR.-82-5096

$6 / 82$

J. R. Ireland THREE MILE ISLAND AND MULTIPLE FAILURE ACCIDENTS

LA-UR-82-3278

$11 / 82$

D. Dobranich STEAM GENERATOR TUBE RUPTURE ANALYSIS FOR TMI-1

LA-UR-82-1786

183

TRAC ANALYSIS OF STEAM-GENERATOR OVERFILL TRANSIENTS FOR TMI-1

LA-UR-81-1230

$4 / 81$

J. R. Ireland THERMAL HYDRAULIC AND CORE DAMAGE ANALYSES OF THE TMI-2 ACCIDENT

LA-UR-80-5044

$10 / 80$ 
Author:

Title:

Report Number: Issue Date:

Author:

Title:

Report Number: Issue Date:

Author:

Title:

Report Number: Issue Date:

Author:

Title:

Report Number:

Issue Date:

Author:

Title:

Report Number:

Issue Date:

Author:

Title:

Report Number:

Issue Date:

Author:

Title:

Report Number: Issue Date:

Author:

Title:

Report Number: Issue Date:

Author:
W. R. Stratton

ALTERNATIVE EVENT SEQUENCES - TECHNICAL STAFF ANALYSIS REPORT ON ALTERNATIVE EVENT SEQUENCES TO PRESIDENT'S COMMISSION ON THE ACCIDENT AT THREE MILE ISLAND

LA-UR-80-1069

$4 / 80$

W. L. Kirchner

FUEL DAMAGE ESTIMATES FOR THE TMI-2 REACTOR

LA-UR-80-0998

$4 / 80$

R. D. Burns

EVALUATION OF THE THREE MILE ISLAND

ACCIDENT IN THE CONTEXT OF W/ASH - 1400

LA-UR-80-0997

$4 / 80$

P. K. Mast

ANALYSIS OF EARLY CORE DAMAGE AT THREE MILE ISLAND

LA-UR-79-2968

$10 / 79$

W. L. Kirchner

FUEL DAMAGE ESTIMATES FOR THE TMI-2

REACTOR

LA-UR-79-2948

$10 / 79$

R. D. Burns

EVALUATION OF THE THREE MILE ISLAND

ACCIDENT IN THE CONTEXT OF WASH - 1400

LA-UR-79-2942

$10 / 7^{\prime 7}$

P. K Mast, et al

ANALYSIS OF EARLY CORE DAMAGE AT THREE

MILE ISLAND

LA-UR-79-2425

$9 / 79$

W. L. Kirchner

PRELIMINARY CALCULATIONS RELATED TO THE ACCIDENT AT THREE MILE ISLAND

LA-TN-MIMAS-82-3

1982

P. Maudlin, et al 
Title:

Report Number:

Issue Date:

Author:

Title:

Report Number: Issue Date:

Author:

Title:

Steady States:

Assessments:

Applications:
DAMAGE ASSESSMENT OF TMI-2

LA-TN-MIMAS-82-1

1982

P. Maudlin, et al

A STUDY OF VESSEL FLOW RESISTANCE FOR TMI-2

NUREG/CR-1353

$3 / 80$

J.R. Ireland, et al

PRELIMINARY CALCULATIONS RELATED TO THE ACCIDENT AT THREE MILE ISLAND

$100 \%$ core power

None

Large Break LOCA

Feed and Bleed Recovery

COMPONENT DESCRIPTION

Core:

6 axial levels, 6 theta segments, 1 radial ring

Vessel:

14 axial levels, 2 radial rings, 6 azimuthal segments, 6 vent valves

Containment:

Balance of Plant:

Primary or Recirc:

Main Steam:

Control Systems:

Auxiliary Systems:

Fuel Rod Behavior:

Kinetics:
Pressure boundary

Feedwater modeled

Both loops modeled in detail

Pressure boundary

Basic plant protection

HPI, LPI, Accumulators

Detailed reflood mode

Input power table only 
PLANT:

Location:

Vendor:

License Date:

Utility:

Type:

MWt::

MWe:

\# of Bundles:

Group \#:

Code:

Q/A:

Organization:

Admin. Contact:

Technical Contact:

\section{REFERENCES}

Report Number:

Issue Date:

Author:

Title:

Steady States:

Assessments:

Applications:

COMPONENT DESCRIPTION

Core:

Vessel:

Containment:

Balance of Plant:

Primary or Recirc:

Main Steam:

Control Systems:

Auxilliary Systems:

Fuel Rod Behavior:

Kinetics:
Tihange 2

Belgium

FRA

$3 / 83$

Societe Intercommunale Belge de Gaz et d'Electricite

3-Loop

2785

941

$157 \quad 17 \times 17$

W6

RELAP5/MOD2

$\mathrm{Y}$

EG\&G Idaho, Inc.
G W Johnsen
208-526-9854
R R Schultz 208-526-9508

NUREG/IA-0044

3/92

G.P. Rouel, et al

Assessment Study of RELAP5/MOD2 Cycle

36.05 Based on the Tihange-2 Reactor

Trip of January 11, 1983

$100 \%$ core power

Plant Trip

None reported
Single channel, five level core

Core bypass, upper head leakage paths

Pressure boundary

Detailed SGs, no feedtrain

3 explicit loops, detailed pressurizer

Detailed steam lines

ECCS, chemical and volume control

None 
PLANT:

Location:

Vendor:

License Date:

Utility:

Type:

MWt:

MWe:

\# of Bundles:

Group \#:

Code:

Q/A:

Organization:

Admin. Contact:

Technical Contact:

\section{REFERENCES}

Report Number:

Issue Date:

Author:

Title:

Report Number:

Issue Date:

Author:

Title:

Report Number:

Issue Date:

Author:

Title:

Report Number:

Issue Date:

Author:

Title:
Vandellos 2

Spain

W

3/88

Asociacion Nuclear Vandellos

3-Loop

2775

982

$\begin{array}{ll}157 & 17 \times 17\end{array}$

W6

RELAP5/MOD2

$\mathrm{Y}$

EG\&G Idaho, Inc.

R R Schultz 208-526-9508

R R Schultz 208-526-9508

ICAP-00215

9/91

C. Llopis

ASSESSMENT OF RELAP5/MOD2 AGAINST A LOAD

REJECTION FROM 100\% TO 50\% POWER IN THE

VANDELLOS II NUCLEAR POWER PLANT

ICAP-00216

C. Llopis

ASSESSMENT OF RELAP5/MOD2 AGAINST A

TURBINE TRIP FROM 100\% POWER IN THE

VANDELLOS II NUCLEAR POWER PLANT

ICAP-00218

9/91

C. Llopis, et al ASSESSMENT OF RELAP5/MOD2 AGAINST A 10\% LOAD REJECTION TRANSIENT FROM 75\% STEADY STATE IN THE VANDELLOS II NUCLEAR POWER PLANT

ICAP-00219

9/91

C. Llopis

ASSESSMENT OF RELAP5/MOD2 AGAINST A MAIN FEEDWATER TURBOPUMP TRIP TRANSIENT IN

THE VANDELLOS II NUCLEAR POWER PLANT 
Steady States:

Assessments:

Applications:

COMPONENT DESCRIPTION

Core:

Vessel:

Containment:

Balance of Plant:

Primary or Recirc:

Main Steam:

Control Systems:

Auxiliary Systems:

Fuel Rod Behavior:

Kinetics:
75\% power; $100 \%$ power; $99.2 \%$ power

Loss of Load

Turbine Trip

Feedwater Transients

None reported
Single channel, 6 node core

Core bypass included

Pressure boundary

Detailed SG secondary, no feedtrain

All 3 loops lumped into 1

Detailed steamline

Rod control, pressurizer level, FW, and turbine and steam dump control

None

None

Point Kinetics 
PLANT:

Location:

Vendor:

License Date:

Utility:

Type:

MWt:

MWe:

\# of Bundles:

Group \#:

Code:

Q/A:

Organization:

Admin. Contact:

Technical Contact:

\section{REFERENCES}

Report Number:

Issue Date:

Author:

Title:

Report Number:

Issue Date:

Author:

Title:

Steady States:

Assessments:

Applications:

COMPONENT DESCRIPTION

Core:

Vessel:

Containment:

Balance of Plant:

Primary or Recirc:

Main Steam:

Control Systems:

Aus:iliary Systems:

Fuel Rod Behavior:

Kinetics:
Vandellos 2

Spain

W

3,88

Asociacion Nuclear Vandellos

3-Loop

2775

982

$\begin{array}{ll}157 & 17 \times 17\end{array}$

W6

TRAC-PF1/MOD1

Y

EG\&G Idaho, Inc.

R R Schultz 208-526-9508

R R Schultz 208-526-9508

ICAP-00175

$9 / 90$

A. Querol, et al

ASSESSMENT OF TRAC-PF1/MOD1 AGAINST A

LOAD REJECTION FROM 100\% TO 50\% IN THE

VANDELLOS II NUCLEAR POWER PLANT

ICAP-00176

$9 / 90$

A. Querol

ASSESSMENT OF TRAC-PF1/MOD1 AGAINST A

TURBINE TRIP FROM 100\% POWER IN THE

VANDELLOS II NUCLEAR POWER PLANT

$100 \%$ power

Loss of Load

Turbine Trip

None reported

Single channel, 6 node core

Core bypass included

Pressure boundary

Detailed SG secondary, no feedtrain

All 3 loops lumped into ?

Detailed steaminire

Rod control, prese level, FW, and

turbine and stean this onst

None

None

Point Kinetics 
PLANT:

Location:

Vendor:

License Date:

Utility:

Type:

MWt:

MWe:

\# of Bundles:

Group \#:

Code:

Q/A:

Organization:

Admin. Contact:

Technical Contact:

\section{REFERENCES}

Steady States:

Assessments:

Applications:

\section{COMPONENT DESCRIPTION}

Core:

Vessel:

Containment:

Balance of Plant:

Primary or Recirc:

Main Steam:

Control Systems:

Auxilliary Systems:

Fuel Rod Behavior:

Kinetics:
Yankee Rowe

Rowe, Mass.

W

$7 / 61$

Yankee Atomic Electric Co.

4-Loop

540

167

$76 \quad 16 \times 16$

W5

RELAP5/MOD3

$\mathrm{N}$

EG\&G, Idaho Inc.

G W Johnsen 208-526-9854

P A Roth 208-526-9188

None

Hot zero power; $100 \%$ core power

None

Small Break LOCA

Single channel, 6 node core

3 downcomer channels, each

split into quarters

Pressure boundary

Simulated main and aux. feed

4 explicit loops

Includes SRVs and TBVs, each line

SG level

SI

None

Power input table 
PLANT:

Location:

Vendor:

License Date:

Utility:

Type:

MWt:

MWe:

\# of Bundles:

Group \#:

Code:

Q/A:

Organization:

Admin. Contact:

Technical Contact:

\section{REFERENCES}

Report Number:

Issue Date:

Author:

Title:

Steady States:

Assessments:

Applications:

\section{COMPONENT DESCRIPTION}

Core:

Vessel:

\section{Containment:}

Balance of Plant:

Primary or Recirc:

Main Steam:

Control Systems:

Auxiliary Systems:

Fuel Rod Behavior:

Kinetics:
Yong-Gwang 2

Korea

W

$6 / 87$

Korea Electric Power Corporation

3-Loop

2785

900

$\begin{array}{ll}157 & 17 \times 17\end{array}$

W6

RELAP5/MOD2

Y

EG\&G Idaho, Inc.

R R Schultz 208-526-9508

R R Schultz 208-526-9508

ICAP-00208

Namsung Arne, et al ASSESSMENT OF RELAP5/MOD2 COMPUTER CODE AGAINST THE NATURAL CIRCULATION TEST DATA FROM YONG-GWANG UNIT 2

$3 \%$ full power

Natural circulation test

None reported

Single channel, six node core

Guide tube flow, core bypass, and

leakage paths modeled

Pressure boundary

No feed train

One double loop, one single loop

Steam lines modeled

Steanı dump

ECCS

None

Pisint Kinetics 
PLANT:

Location:

Vendor:

License Date:

Utility:

Type:

MWt:

MWe:

\# of Bundles:

Group \#:

Code:

Q/A:

Organization:

Admin. Contact:

Technical Contact:

\section{REFERENCES}

Report Number:

Issue Date:

Author:

Title:

Steady States:

Assessments:

Applications:

COMPONENT DESCRIPTION

Core:

Vessel:

Containment:

Balance of Plant:

Primary or Recirc:

Main Steam:

Control Systems:

Auxiliary systems:

Fuel Rod Behavior:

Kinetics:
Zion 1

Zion, Ill.

W

$12 / 73$

Commonwealth Edison Co.

4-Loop

3250

1040

$19315 \times 15$

W3

RELAP5/MOD2

Y

EG\&G Idaho, Inc.
G W Johnsen
208-526-9854
C B Davis
208-526-9470

NUREG/CR-4672

12/86

C. D. Fletcher

ANALYSIS OF INSTRUMENT TUBE RUPTURES IN WESTINGHOUSE 4-LOOP PRESSURIZED WATER

REACTORS

100\% Power/ 100\% Flow

None

Instrument Tube Ruptures

Six node core, Six node bypass

4 node lower plenum, 14 node downcomer,

10 node upper plenum/upper head

Pressure boundary

Feedwater modeled

3 primary loops lumped into 1 , plus one

single loop. 17 nodes per loop.

Pressure boundary

None

auxiliary feedwater, LPI,HPI,

accumulators

None

Input power table only 
PLANT:

Location:

Vendor:

License Date:

Utility:

Type:

MWt:

MWe:

\# of Bundles:

Group \#:

Code:

Q/A:

Organization:

Admin. Contact:

Technical Contact:

\section{REFERENCES}

Report Number:

Issue Date:

Author:

Title:

Report Number:

Issue Date:

Author:

Title:

Report Number:

Issue Date:

Author:

Title:

Renort Number:

Issue Date:

Author:

Title:

Report Number:

Issue Date:
Zion 1

Zion, Ill.

W

$12 / 73$

Commonwealth Edison Co.

4-Loop

3250

1040

$19315 \times 15$

W3

TRAC-PF1/MOD1

Y

Los Alamos National Laboratory

B B Boyack 505-667-4097

B B Boyack 505-667-4097

NUREG/CR-2656

$5 / 82$

N.S. DeMuth, et, al

LOSS-OF-FEEDWATER TRANSIENTS FOR THE

ZION-1 PRESSURIZED WATER REACTOR

LA-UR-84-3083

1984

B. D. Boyer

AN ASSESSMENT OF THE UTILITY OF

FEED-AND-BLEED OPERATING MAP (ZION-1)

LA-UR-83-3376

1983

B. E. Boyack, COMBINED MAIN FEED LINE BREAK/ LOSS-OF-FEEDWATER TRANSIENT IN THE ZION-1 PWR

LA-UR-83-3251

1983

B. Nassersharif COMBINED STEAM-GENERATOR-TUBE-RUPTURE AND LOSS-OF-FEEDWATER TRANSIENTS FOR THE ZION-1 PWR

LA-UR-83-2787

1983 
Author:

Title:

Report Number:

Issue Date:

Author:

Title:

Report Number:

Issue Date:

Author:

Title:

Report Number:

Issue Date:

Author:

Title:

Report Number: Issue Date:

Author:

Title:

Report Number:

Issue Date:

Author:

Title:

Report Number:

Issue Date:

Author:

Title:

Report Number:

Issue Date:

Author:

Title:
B. E. Boyack

COMBINED MAIN-STEAM-LINE-BREAKJ

LOSS-OF-FEEDWATER TRANSIENTS IN THE

ZION-1 PWR

LA-UR-83-2684

1983

B. E. Boyack

SHUTDOWN TO RESIDUAL HEAT REMOVAL COOLING IN THE ZION-1 PWR FOLLOWING A SEVERE. LOSS-OF-FEEDWATER EVENT

LA-UR-83-i 943

1983

C. Watson, et al

STEAM-GENERATOR-TUBE-RUPTURE WITH

OPERATOR ACTION ANALYSIS FOR ZION-1

LA-UR-83-2224

1983

B. Boyack LOSS-OF-FEEDWATER TRANSIENTS FOR THE ZION-1 PWR

LA-UR-83-1731

$6 / 83$

B. Boyack SEVERE LOSS-OF-FEEDWATER TRANSIENTS IN THE ZION-1 PWR

LA-UR-83-1714

1983

B. Boyack LOSS-OF-OFFSITE POWER TRANSIENT FOR THE ZION-1 PWR ANS PAPER

LA-UR-82-3713

$12 / 82$

C. E. Watson STEAM GENERATOR TUBE RUPTURE WITH OPERATOR ACTION ANALYSIS FOR ZION-1

LA-UR-82-3236

1982

D. Dobranich

STEAM GENERATOR TUBE RUPTURE ANALYSIS

FOR ZION-1 
Report Number:

Issue Date:

Author:

Title:

Report Number:

Issue Date:

Author:

Title:

Report Number:

Issue Date:

Author:

Title:

Report Number:

Issue Date:

Author:

Title:

Report Number: Issue Date:

Author:

Title:

Report Number:

Issue Date:

Author:

Title:

Report Number:

Issue Date:

Author:

Title:
LA-UR-82-1333

1982

N. S. DeMuth

LOSS-OF-FEEDWATER TRANSIENTS FOR THE

ZION-1 PRESSURIZED WATER REACTOR

LA-UR-85-0858

3/85

R. Fujita, et al

TRAC-PF1 ANALYSIS OF A BEST-ESTIMATE

LARGE-BREAK LOCA IN A WESTINGHOUSE PWR

WITH FOUR LOOPS AND 17X17 FUEL

LA-UR-84-2056

1984

J. F. Lime

LOSS-OF-FEEDWATER/FEED AND BLEED IN A

WESTINGHOUSE THREE-LOOP, LOW-HEAD SAFETY

INJECTION PWR

LA-UR-84-0109

$1 / 84$

E. Barts, et al TRAC.PF1 STUDY OF LOSS OF PUMP SEALS IN A WESTINGHOUSE PWR

LA-UR-85-3117

$8 / 85$

R. P. Jenks, et al PRELIMINARY TRAC CALCULATION OF R. E. GINNA STEAM GENERATOR TUBE-RUPTURE EVENT ON JANUARY 25, 1982

LA-UR-82-3237

$11 / 82$

J. F. Lime

TRAC-PD2 CALCULATIONS OF A

MAIN-STEAM-LINE BREAK IN A WESTINGHOUSE

PRESSURIZED WATER REACTOR-COOLANT PUMP

TRIP

LA-UR-82-3235

$11 / 82$

J. F. Lime

TRAC-PD2 CALCULATION OF A COLD-LEG SMALL

BREAK IN A WESTINGHOUSE FOUR-LOOP

PRESSURIZED WATER REACTOR 
Report Number: Issue Date:

Author:

Title:

Report Number: Issue Date:

Author:

Title:

Report Number: Issue Date:

Author:

Title:

Report Number: Issue Date:

Author:

Title:

Report Number: Issue Date: Author:

Title:

Report Number:

Issue Date:

Author:

Title:
NUREG/CR-3312

$5 / 83$

J. E. Elliott

EFFECTS OF THE REACTOR COOLANT PUMPS

FOLLOWING A SMALL BREAK IN A

WESTINGHOUSE PWR

LA-UR-85-4145

1985

Bahram Nassersharif

ANALYSIS OF STEAM GENERATOR TUBERUPTURE EVENTS CCMBINED WITH AUXILIARYFEEDWATER CONTROL_SYSTEM FAILURE FOR THE THREE MILE ISLAND, UNIT 1 AND ZION, UNIT I PRESSURIZED WATER REACTOR

LA-UR-85-2816

1985

Bahram Nassersharif

ANALYSIS OF STEAM GENERATOR TUBERUPTURE EVENTS COMBINED WITH AUXILIARYFEEDWATER CONTROL_SYSTEM FAILURE FOR THREE MILE ISLAND, UNIT 1 AND ZION, UNIT 1 PRESSURIZED WATER REACTORS

LA-UR-85-1773

1985

Bahram Nassersharif

ANALYSIS OF STEAM GENERATOR TUBERUPTURE EVENTS COMBINED WITH AUXILIARYFEEDWATER CONTROL-SYSTEM FAILURE FOR THREE MILE ISLAND, UNIT 1 AND ZION, UNIT 1 PRESSURIZED WATER REACTORS

LA-UR-84-3221

1984

B. Nassersharif, et al UNMITIGATED BORON DILUTION EVENTS IN OCONEE - 1, CALVERT CLIFFS, AND ZION - 1 PRESSURIZED WATER REACTORS DURING SHUTDOWN

LA-UR-82-1821

$6 / 82$

James F. Lime

TRAC/PD2 MODELING OF A COLD-LEG

0.0002 M2 BREAK IN BABCOCK AND WILCOX, 
COMBUSTION ENGINEERING, AND WESTINGHOUSE PRESSURIZED WATER REACTORS

Steady States:

$100 \%$ core power

Assessments:

None

Applications:

Feedwater Transients

\section{COMPONENT DESCRIPTION}

Core:

Vessel:

Containment:

Balance of Plant:

Primary or Recirc:

Main Steam:

Control Systems:

Auxiliary Systems:

Fuel Rod Behavior:

Kinetics:
4 levels, 2 theta, 1 radial

10 levels, 2 theta, 2 radial

Pressure boundary

Detailed secondary

Three loops modeled as one, pressurizer loop in detail

Steam lines modeled; SRV and ARVs are

modeled. Steam generators modeled; no

PORV, SRV, PRT, ARV

ECCS system

Detailed rod conductive and reflood

heat transfer

Input power table only 


\section{Appendix B}

Using the Database Programs

B-1 


\section{Appendix B}

\section{Using the Database Programs}

\section{B.1 The Data Input Program}

This program allows editing and appending of data into the primary database files: the plant information, report information, and deck information files. At the main menu the user may select the database file to be edited. Once this has been done the user is viewing the records from the chosen database and has the option of editing existing records, inserting or appending new records, deleting records, and moving through the database file. Selections are made from the prompt at the bottom line of the screen by typing the letter which designates the desired action.

\section{B.2 The Query Program}

The query program is a user friendly program for extracting information from the database. Search parameters are selected from menus so the typing is minimal, and typing errors are eliminated.

The database may be searched by specifying the desired search parameters with the SELECTION option and letting the program narrow down the full database until only those entries which satisfy all the parameters remain. The intermediate lists of plant decks which are generated along the way are accessible to the user by using the PREYIOUS and NEXT features to move between lists; if the final list is unsatisfactory you can back up to a more general, more extensive list.

The user may specify any of the plant categories (i.e. plant name, group, ype or vendor), the modeling code, or the application (such as a large break LOCA), when using the selection option. Any combination of one plant category, an application, and a code may be specified. Plant categories above the chosen category in the hierarchy are automatically supplied by the program, those below are left at the default value of "Any" which informs the program to ignore those parameters when conducting the search. For convenience, the categories group, type, and vendor may be set by specifying a plant name from that category. For example, if you are interested in finding models for plants similar to Wolf Creek you may set the group, type or vendor parameter by choosing the name Wolf Creek in response to the "By Plant" option, and then carry out the search. Even if the user knows nothing about the plant whose deck he needs to locate, beyond the name of the plant, he can easily find if that deck is available. If it is not, the program will automatically locate the best alternative decks for plants of a similar group, type, or vendor, in that order.

The user should leave the SELECTION option by choosing "Go" after setting the search parameters. "Go" initiates the automatic search; "Exit" aborts the SELECTION option. In either case control returns to the main menu. SHOW will display the list of plant decks found by the search 
and allow the user to scroll through the list of decks and choose one to look at in more detail. All the data about the plant deck will be displayed. A report may also be sent to a printer or file if the user desires.

Menus, and messages, combine to make the program easy to use. 

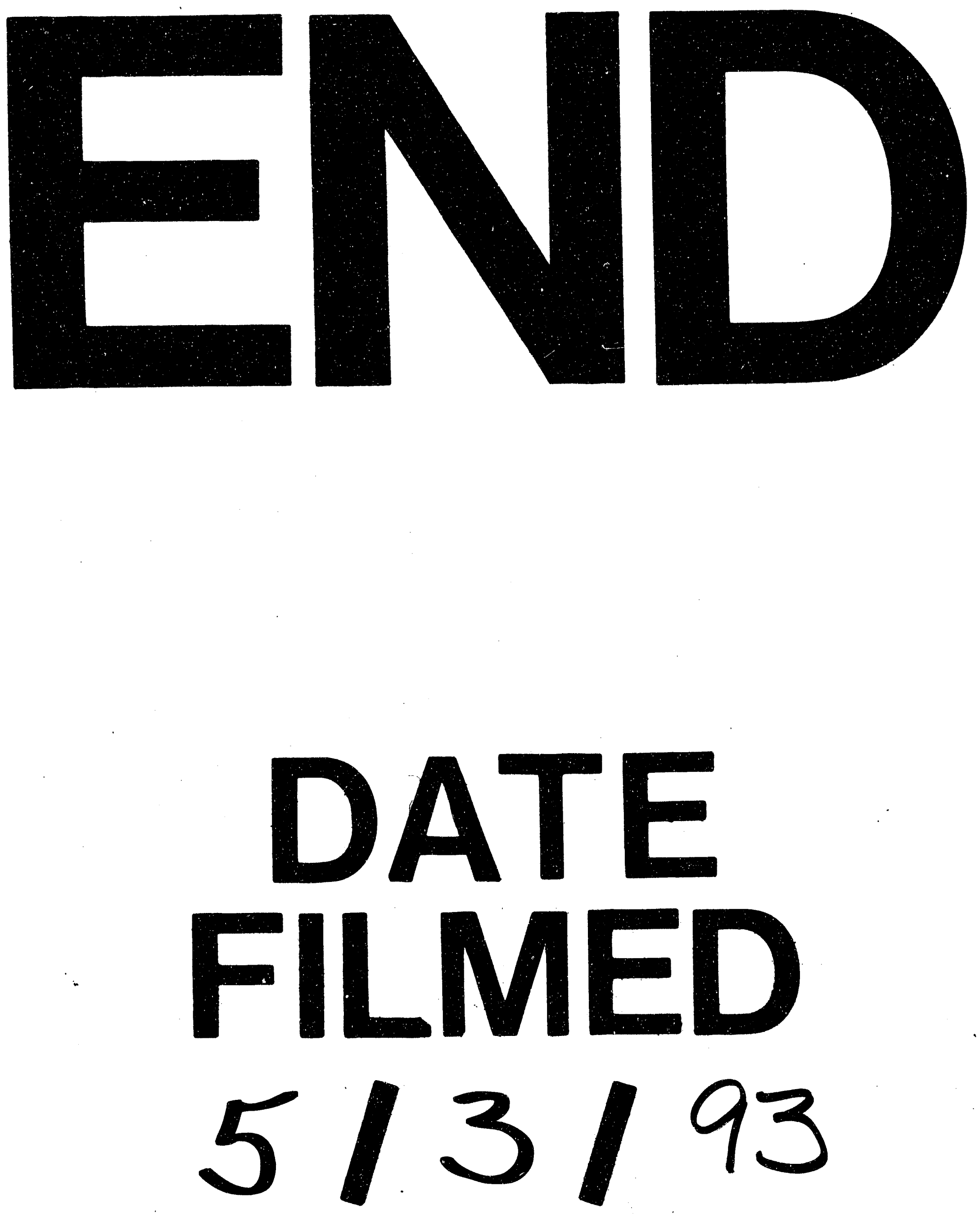
